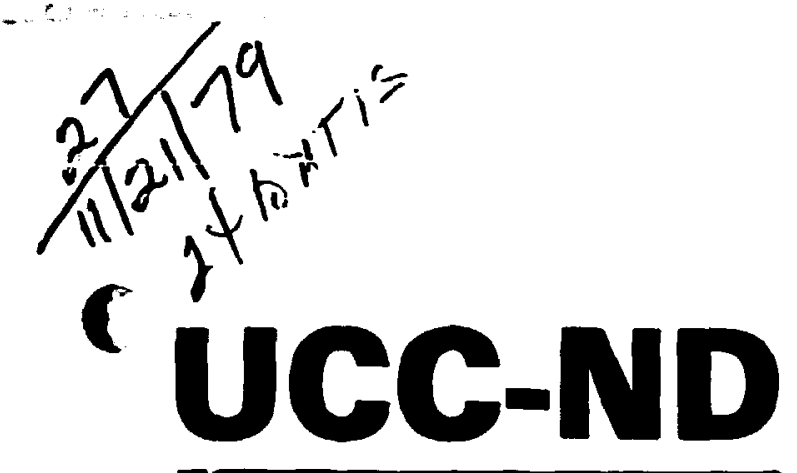

NUCLEAR DIVISION

UNION CARBIDE

\title{
ORNL/CSD/TM-93
}

\section{Information and Advice on the Numerical Software Available for the Fusion Energy Program at Oak Ridge}

P. W. Gaffney

GPFEAIEO GY

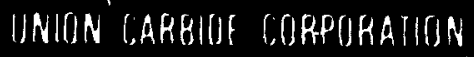

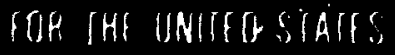


Contract No. W-7405-enz-26

COMPUTER SCIEACES DIVISICN

IMFORATION AND ADVICE ON THE NUMERICAL SOFTWARE AVAILABLE FOR THE FUSION ENERGI PROGRAY AT OAR RIDGE

P. W. Gaffney

Sponsor: R. A. Dory

Originator: P. W. Gaffney

Date Published - November 1979

NOTICE

This document contains information of a preliminary nature. It is subject to revision or correction and therefore does not represent a final report.

UNION CARBIDE CORPORATION, NUCLEAR DIVISION Operating the

Oak Ridge Gaseous Uiffusion Plant - Oak Ridge National Laboratory

Oak Ridge Y-12 Plant Paducah Gaseuus Diffusion Plant for the

DEPARTMENT OP ENERGY

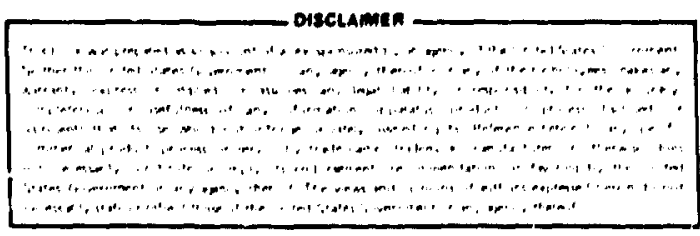


iii

BEABASK DHTE: SEptember 1979 
TAE:-

ACRYOULDGMENTS ...................... vii

ABSTRACT .......................... 1

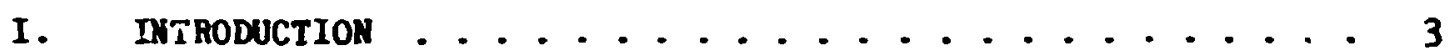

II. PROBlEM AREAS .................... 7

III. SOETWAPE CONTENTS . . . . . . . . . . . . 9

IV. ABBREVIATIONS USED .................... 11

v. SOFTWAE .................... iz

AFPEUDXX A: hvailability of Software ............. 69

APPENDIX B: The MASTI Database ............... 73

APPENDIX C: SANDIA Special Sunction koutines . . . . . . . 79

APPENDIX D: The Contents of MP ............. 83 


\section{ACRYOULEDGHENTS}

I wish to thank Richard Hicks and Bill van Rij for making sereral suggestions which have led to improvements in this report. Horeover, I also extend ay thanks to Janice Hughes for her patience and diligence throughout the typing af this report.

Supinrt for this work was provided by the Fuston Energy Division and the Computing Applications Department, Computer Sciences Division. 
The purpose of this report is to describe some of the numerical routines that have been rotained for the Fusion Energy progran at Oak Ridge.

The report is organized by problem area. Each area contains a list of relevant numerical routines. These routines are described in detail and, where appropriate, we give advice on their correct use. Furtherwore, we have ranked the subroutine libraries MAG, IMSL, and HARNEL according to how satisfaztory their routines are for that particular area.

In an Appendix we provide information on how to access the routines and the subroutine libraries described in this report. Moreover, we describe a growing online data bise which contains a condensed version of the inforiation in this report. 


\section{INTRODUCTION}

The purpose of this report is to describe some of the wathematical software that has been obtained for the Fusion Energy program at Oak Ridge. However, because we provide state-of-the-art numerical support for tisis program, the report is of necessity i!lcomplete.

The repret is designed in such a way that a computer user may conveniently obtain information and advice on the use of the numerical routines. Thus, in Section $v$, each routire has been placed in a particular problem area. A list of the 21 problem areas that are covered by this report is given in section II. At the beginning of each problem area, we list the major subrout.ine libraries which include software that we. :ommend for that particular area. The subroutine libraries that we have considered are NAG, IMSL and HARWELL, and the order in which they are presented within each problem area reflects the author's recommendations.

In addition to the software contained in the above subroutine libraricis, there exists a wide variety of numerical routines for the problem areas covered by this report. However, some of these routines fall to teet our requirement of being state-of-the-art. Therefore, for some problem areas there are few routines that ke can recommend; and in this case we rely upon the above subrolitine libraries to provide the appropriate codes. When state-or-the-art software exists at Oak Ridge, we have endeavored to include a description of it. in this report. However, since the phrase "state-of-the-art," is open to interpretation, some people may 

disagree with the author's selection of routines. Furtherwore, since this report currently coicains information on only 79 routines, it follows that we have not described all state-of-the-art sortware in Oak Ridge. To inform users of new software and to nininize the number of quality routines that are onitted from the report, we intend to provide updates at regular intervals.

$T^{2}$ : description of the additional software is presented in the form:

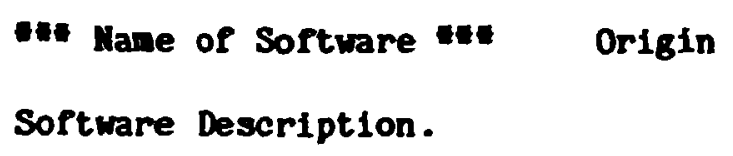

We have not attenpted to rank this software. However, in most cases have included in the description some advice on the use of the software. Usually, this advice is given on the basis of our experience with the software.

In Appendix $A$, we describe the avallability of the software to computer users at Oak Ridge. In Appendix B, we describe a growing online data base, called MASTI (Numerical Analysis Software Tutorial Inquirer), which is designed to inform computer users of some of the state-of-the-art mathematical routines that exist at Oak Ridge. The information contained in this report is avallable in a condensed form in MASTI.

In Appendix C we give a list of the subroutines in the Sandia Mathematical Subroutine Library that may be used for approximating special functions. In Appendix D, we present the contents of 
Richard Brent's nultiple precision package. We believe that this package will be very useful to conputer users.

We have prepared this report using IBDI. In this way the report can be easily updated and copies nay be readily obtained. To ensure that users are referring to the nost recent issue of this report, we have provided at the front of the report a release date for each update. When updates are made, the release date will change accordingly and a copy of the new report will be available on microfiche from the author.

This is a text erfior/formatter developed by Clair Ne1lson, Los Alamos Scientific Laboratory. 
II. PROBIOY AREAS

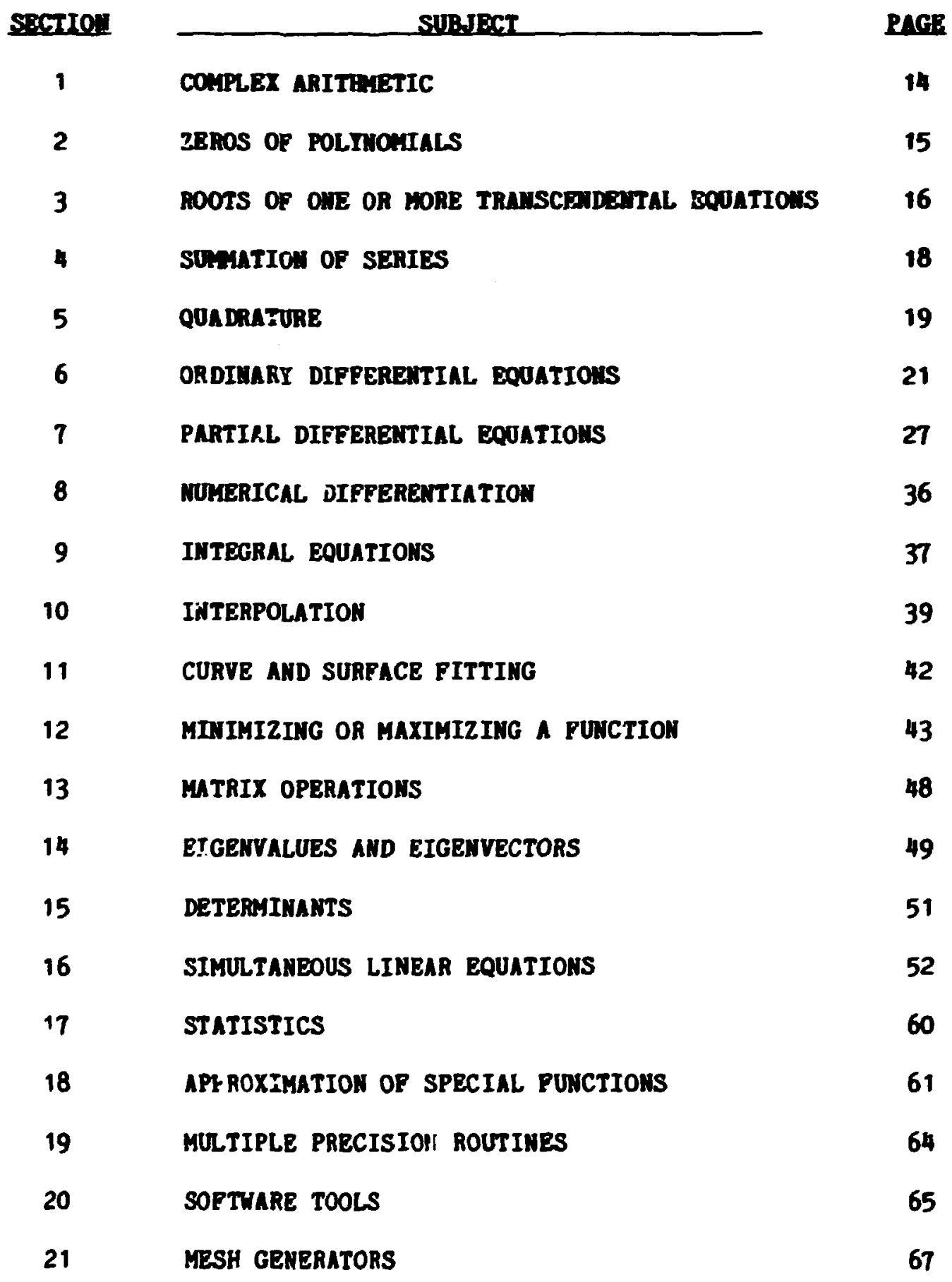


IIT. SCETHARE COATETS

\begin{tabular}{|c|c|c|c|}
\hline IITLE & PAGE & TITLE & PAGE \\
\hline M02MAF & 14 & FTTPAK & 18 \\
\hline A02ABF & 14 & FMIN & 45 \\
\hline A02ACF & 14 & FUNPACK & 61 \\
\hline ADMPT & 42 & GEAR & 23 \\
\hline BICUBIC & 40 & GEARB & 23 \\
\hline BLKTRI & 29 & GEARBD & 23 \\
\hline BRENT 1 & 15 & GEARBI & 23 \\
\hline BRNANL & 66 & GEARBIL & 23 \\
\hline BT & 55 & GEARIB & 23 \\
\hline BTMS & 55 & CEARS & 23 \\
\hline CHES & 58 & GERK & 21 \\
\hline -OLG.JE & 24 & GMA & 57 \\
\hline DAVE & 65 & GMAS & 57 \\
\hline D04AAF & 36 & HYBRD1 & 17 \\
\hline D05AAF & 37 & IDBVIP & 42 \\
\hline D05ABF & 37 & IDSPFT & 42 \\
\hline DE & 22 & LAMSO & 50 \\
\hline DECOMP & 52 & LANSOI & 50 \\
\hline DEROOT & 22 & LAHSON-HANSON CODES & 58 \\
\hline EISPACK & 49 & LINPACK & 53 \\
\hline BPISODE & 24 & LMDER 1 & 43 \\
\hline EPISODEB & 24 & LMDIF 1 & 4 \\
\hline
\end{tabular}




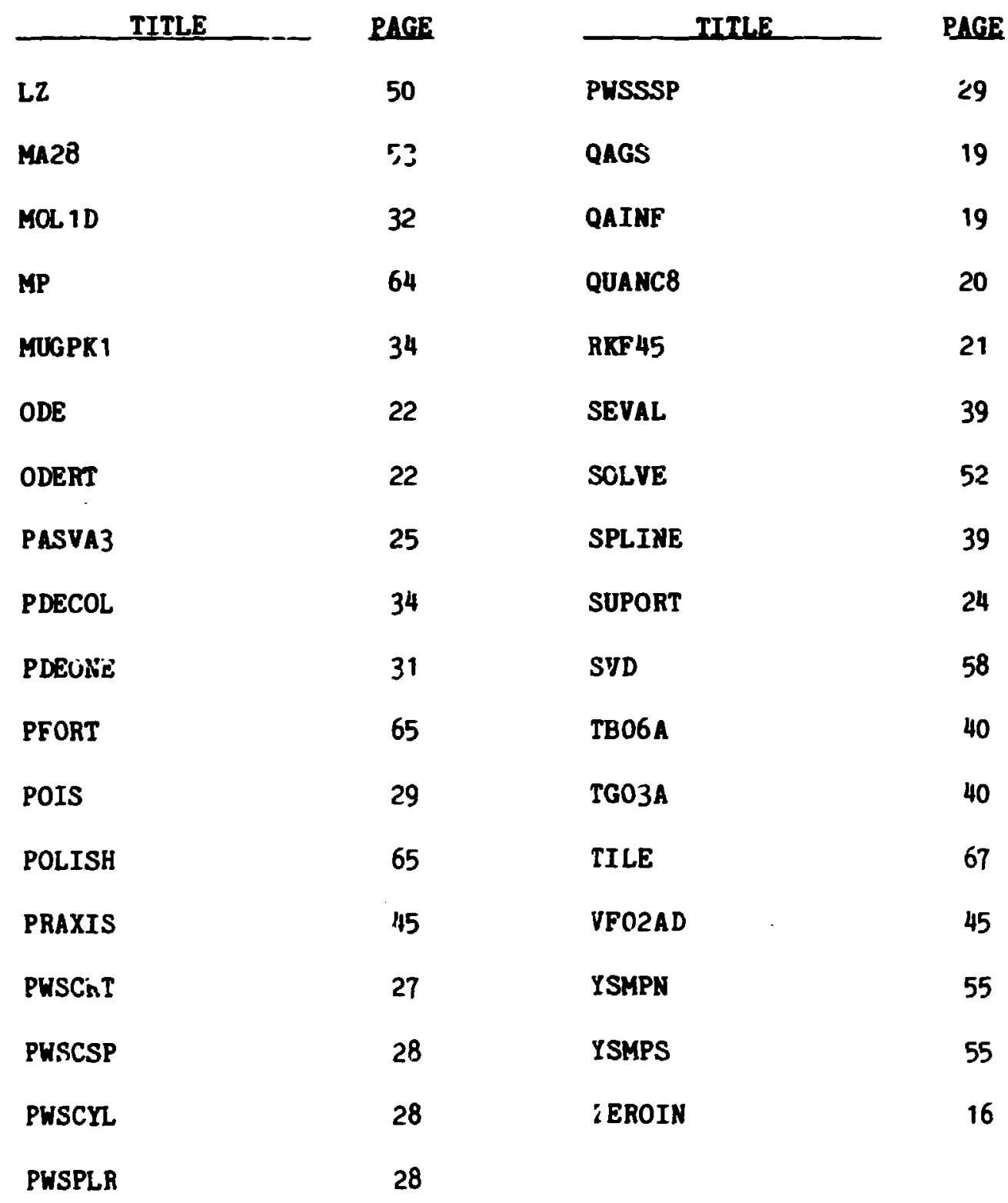


IV. ABBREVIATIOUS USED

Abbreviation

ACH .LLL

FTy

HARNELL

IMSL

MINPACK1

NAG

NCAR

QUADPACK

S.G.

S.S.

TOMS Mean:ing and Information

Alan C. Hindmarsh, Liurence Livermore Laboratory.

"Computer Methods for Mathematical Computations" by G. E. Forsythe, M. Malcolm and C. B. Moler. Psblished by Prentice-Hall.

Harwell Subroutine Library.

International Mathematical and Statistical Libraries.

A subroutine package for optimization. The package is being constructed at the Argonne National Laboratory by B. S. Garbow, K. E. Hillstrom and J. J. More.

Numerical Algorichms Group.

National Center for Atmospheric Research.

A subroutine package for numerical integration. The package is being constructed in a joint profect by the "Technische Universi'usi. Hien" (H. J. Stetter and C. W. Uberhuber, Vierna, Austria) and the "Katholiel:e Universiteit Leuven" ( $n$. Piessens and $E$. de Doncker, Leuven, Belgium).

"Computer Solution of Ordinary Differential Equations" by L. F. Shampine and M. K. Gordon published by $X$. H. Freeman and Company.

"Efficient FORTRAN Subprograms for the Solution of Elliptic Partial Differential Equations," Oy $P$. Swarztrauber and R. Sweet, NCAR Technical Note/IA-.109, July 1975

ACM Transactions on Mathematical Software. 
Y. SOETIARE 


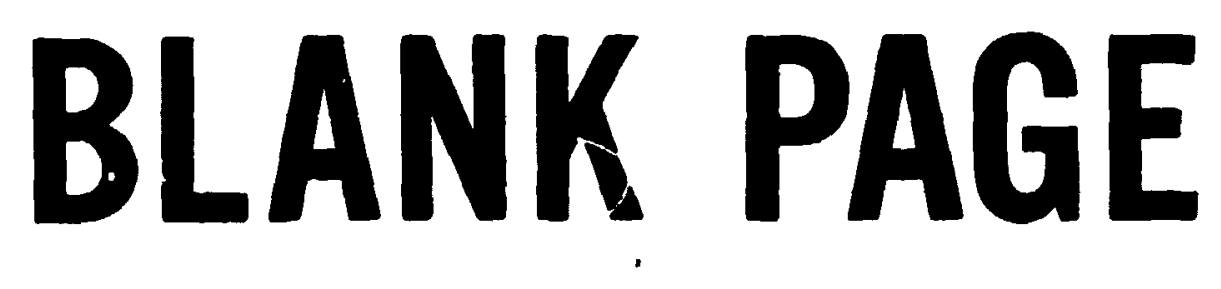




\section{COYPLET ARITHOMETIC}

NAG

The 102 chapter of the MAG slibroutine library contains three sutroutines for arithmetic operations involving complex numbers. They are:

A02AF Evaluates the sonuare root of a complex number.

102ABF Evaluates the modulus of a complex number.

AC2ACF Divides one complex number by a second complex number.

These routines were originally written for usz by the NAG library routines which compute eigensystems of real and complex matrices. However, they may be of general use to programers using complex numbers. 


\section{TEROS OF POLIIIOMTHS}

IMSL

HARNEL

NAG

The appropriate routines in the above libraries are recomended. 


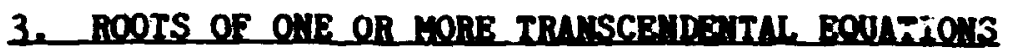

MAG

BARUEL

IISL

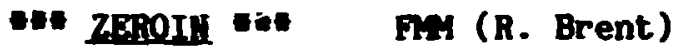

This is a function subprogran for computing a real zero of a single function. That is, find $x$ such that

$$
f(x)=0 \text {. }
$$

The numerical method that is used is a combination of bisection, inverse quadratic interpolation aid the secant method. ZEROIN is reliable, fast and accurate. Therefore, it is highly recomended.

\section{WE BRENT *he MINPACK1}

This subroutine tries to find a solution to a system of $\mathrm{N}$ nonlinear equations in $N$ unknowns. That is, EkENT 1 tries to find $x_{1}, \ldots, x_{N}$ such that

$$
\begin{gathered}
f_{1}\left(x_{1}, \ldots, x_{N}\right)=0 \\
f_{2}\left(x_{1}, \ldots, x_{N}\right)=0 \\
\vdots \\
f_{N}\left(x_{1}, \ldots, x_{N}\right)=0 .
\end{gathered}
$$

The numerical method that is used is due to $R$. Brent. This routine is not as eliable as the following routine HYBRD1. However, on those p:oblens for which it is successful BRENT 1 works extremely well a'd is very fast. 
Interested users are referred to the paper, Humerical Solution of Monlinear Equations" by Jorge J. More and Michael Y. Cosnard, which was published in Tors, March 1979, Vol. 5, No. 1, pp. 64-85.

\section{*E: HYARD1 HE MINPACK1}

This subroutine tries to find a solution to a system of N nonlinear equations in $\mathrm{N}$ unknowns by a modification of the Powell method. The routine is reliable and accurate, and it has worked successfully on a large number of problems. Thus it is recommended without hesitation. 


\title{
4. SUYWTIOH OF SERIES
}

\section{MAG}

HARIELL

IMSL

\begin{abstract}
-eE ETPAK tee MCAR
A package of Fortran subroutines for the Fast Fourier Transfore of (Real or Complex) periodic sequences and other symetric sequences.
\end{abstract}




\title{
5. OUADRATURE
}

\section{MAG}

\section{HARWELL}

IMSL

\begin{abstract}
Adaptive quadrature routines should be used with a great deal of caution. In particular if the integrand $f(x)$ possesses a derivative which is unbounded, then an adaptive quadrature routine may prove too expensive and may produce an inaccurate answer. In general any properties of $f(x)$ should be fully exploited by the integration routine. This often means that adaptive routines are excluded from considerátion.
\end{abstract}

\section{- oAgS. QAIRE wa (from QUADPACK)}

QACS and QAINF are two s!!s'outines for automatic integration. Specifically, QAGS calculaces an approximation to the integral

$$
\int_{a}^{b} f(x) d x
$$

where $a$ and $b$ are sinite numbers, and QAINF calculates an approximation to integrals of the form

$$
\int_{a}^{\infty} f(x) d x, \quad \int_{-\infty}^{b} f(x) d x, \quad \int_{-\infty}^{\infty} f(x) d x .
$$

The subroutines are globally adaptive, and they each use a non-linear extrapolation technique that is based on the epsilon algorithm. Because of their robustness, they are capable of 
integrating functions which have jump discontinuities and singularities. QAGS and QAINF have worked very well on a wide selection of test problems, and they have proved to be economical in function evaluations, accurate and fast.

The routines QAGS and QAINF are part of the integration package called QHADPACK which is being developed jointly at the 'Technische Universitat Wien' in Austria and the 'Katholieke Universiteit Leuren' in Beisium.

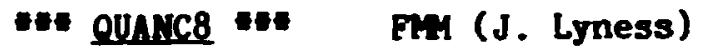

This is an adaptive quadrature routine which tries to approximate the integral

$$
\int_{a}^{b} f(x) d x
$$

to within a user specified tolerance. The limits $a$ and $b$ must be finite. The numerical method that is used is an adaptive Newton Cote's 8-panel rule. Because QUANC8 endeavors to be reliable and because of the adaptive technique that it employs, it is possible that a large number of function evaluations may be used to obtain the required accuracy. 


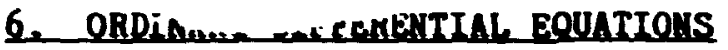

HAG

IMSL

\section{Initial Value Problems}

The following codes integrate a system of $\mathrm{n}$ first ord sr ordinary differential equations:

$$
\frac{d y_{1}}{d t}=f_{i}\left(t, y_{1}, \ldots, y_{n}\right) \quad i=1, \ldots, n
$$

given initial values of $y_{1}, \ldots, y_{n}$. The codes differ in the numerical methods that are used to apr،oximate the solution of the differential equations. Therefore, some advice is given on the types of problems that each code is suitable for.

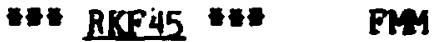

This code uses the Runge-Kutta-Fehlberg (4-5) method with stepsize control.

If the functions $f_{i}\left(t, y_{1}, \ldots, y_{n}\right), i=1, \ldots, n$ are not too expensive to evaluate, and if fairly modest accuracy is requested (for example less than 8 significant digits), then RKF45 is appropriate.

- GERK *W. L. F. Shampine and H. A. Watts

Uses the Runge-Kutta-Fehlberg (4-5) method with stepsize control and global error assessment.

GERK provides a reliable and efficient estimate of the global error, that is, the difference between the solution of the problem posed and the numerical result returned by the code. Therefore, 
if it is important to have a readily available estimate of the actual global error comitted (for exanple in those cases where the differential equations be mathematically unstable), then GERK should be used.

\section{-HE DE, ODE *E: S.G}

Uses a variable order Adans nethod to dynanically adapt the order of integration to the problem. The main difference between $D E$ and $O D E$ is in the number of differential equations that each can integrate. $D E$ has been coded to integrate up to 20 first order differential equations, whereas ODE has no such restriction.

If the enctions $f_{i}\left(t, y_{1}, \ldots, y_{n}\right), i=1, \ldots, n$ are expensive to evaluate, and if high accuracy is required, then DE or ODE is appropriate. If the equations are stiff, then subroutine GEAR should be used. However, for non-stiff problems DE (or ODE) is recommended in preference to using GEAR with the non-stiff option.

\section{* DEROOT, ODRAT *E S.G}

These codes use the same numerical method as $D E$ and $O D E$ to solve the differential equations. In addition DEROCT and ODERT have been designed to compute a root of the user-specified nonlinear scalar equation

$$
B\left(t, y_{1}, \ldots, y_{n}\right)=0 \text {. }
$$

They should be used only when the application calls for a root-finding feature which depends upon the solution of the initial value problem. The main difference between DEROOT and ODERT is that DEROOT has been coded to integrate up to 20 first 
order differential equations whereas the naximun nuaber of equations that ODERT will integrate is user supplied.

\section{** GEAR uE ACH.LLL}

Jses variable order Adans method and backward differentiation formulas. It is particularly suitable for stiff systens bit it can be used for non-stiff systems. However, DE (or ODE) is preferable for non-stiff problems. When the differential equations are stit $f$, the methods used by GEAR require the Jacobian matrix:

$$
\left[\frac{\partial f_{i}}{\partial y_{j}}\right]
$$

The user miy supply this atrix or alternatively it can be approximated by the routines in GEAR.

GEARB: Sing: $\geq$ precision version for banded Jacobian matrices.

GRARBD: Double precision version of GEA:-9.

GEARBI: Useful for ordinary differential equations which arise from the method of lines solution of two- or three-dimensional P.D.E.'s.

GRARBIL: Large Core version of GEARBI.

GRARIB: Often a system of ODE's is not given explicitly, but is given in an implicit form, e.g., $A(y, t) \dot{y}=g(y, t)$, where $A$ is an $n \times n$ matrix. The variant GEARIB was written to solve this class of problems, where the matrices $A$ and $\partial g / \partial y$ are banded.

GEARS: A variant which is useful for problems which involve a general sparse Jacobian matrix. 
EPTSODE: A package of routines which use variable step size formulas. These routines differ from those in the GEAR package in that the latter are based on fixed stsp size fr:mulas, with step changes achieved by interpolation. For problems that require frequent and drastic step size changes, EPISODE is likely to be Dore efficient than GE. ?.

EPISODER: The only variant, so far, of EPISODE. It is intended for banded Jacobıan matrices.

WE COLODE * . B. L. Hulme and S. L. Daniel

Uses collocation methods to solve stiff ordinary differential equations. Because of high overhead, COLODE is generally inerficient for non-stirf problems.

Boundary Value Problems

SUPORT M. R. Scott and H. A. Watts

This code solves a linear two-point boundary value problem of the form

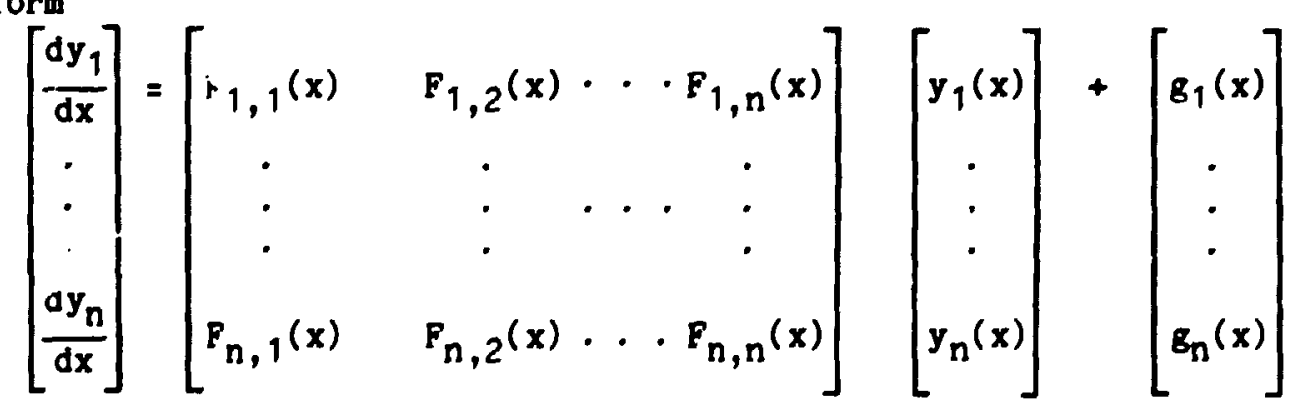

subject to the boundary conditions 


$$
\left[\begin{array}{ccc}
a_{1,1} & a_{1,2} & a_{1, n} \\
\vdots & \vdots & \vdots \\
a_{n-k}, 1 & a_{n-k, 2} & a_{n-k, n}
\end{array}\right]\left[\begin{array}{c}
y_{1}(a) \\
\vdots \\
y_{n-k}(a) \\
\vdots \\
y_{n}(a)
\end{array}\right]=\left[\begin{array}{c}
a_{1} \\
\vdots \\
a_{n-k}
\end{array}\right] \quad 1 \leq k \leq n-1
$$

and

$$
\left[\begin{array}{ccc}
b_{1,1} & b_{1,2} & b_{1, n} \\
\vdots & \vdots & \vdots \\
b_{k, 1} & b_{k, 2} & b_{k, n}
\end{array}\right]\left[\begin{array}{c}
y_{1}(b) \\
\vdots \\
y_{k}(b) \\
\vdots \\
y_{n}(b)
\end{array}\right]=\left[\begin{array}{c}
\beta_{1} \\
\vdots \\
\beta_{k}
\end{array}\right]
$$

where the matrix $A=\left[a_{i, j}\right]$ is of rank $n-k$ and the matrix $B=$ $\left[b_{i, j}\right]$ is of rank $k$.

SUPORT uses the method of surerposition together witn orthonormalization. This results in a very reliable and efficient code for solving linear differential equations. The code is primarily intended for solving problems which may be classtified as mathematically unstable, that is, problems with e:ponentially growing solutions.

\section{**ASVA3 * M. Lentinif and V. Pereyra}

This code solves nonlinear two-point boundary value problems of the form 


$$
\begin{aligned}
& \frac{d y_{1}}{d x}=f_{1}\left(x, y_{1}, \ldots, y_{n}\right) \\
& \frac{d y_{2}}{d x}=f_{2}\left(x, y_{1}, \ldots, y_{n}\right) \quad a \leq x \leq b \\
& \vdots \vdots \\
& \frac{d y_{n}}{d x}=f_{n}\left(x, y_{1}, \ldots, y_{n}\right)
\end{aligned}
$$

subject to

$$
\begin{gathered}
B_{1}\left(y_{1}(a), \ldots, y_{n}(a), y_{1}(b), \ldots, y_{n}(b)\right)=0 \\
B_{2}\left(y_{1}(a), \ldots, y_{n}(a), y_{1}(b), \ldots, y_{n}(b)\right)=0 \\
\vdots \\
B_{n}\left(y_{1}(a), \ldots, y_{n}(a), y_{1}(b), \ldots, y_{n}(b)\right)=0 .
\end{gathered}
$$

It uses a variable order finite difference method combined with deferred corrections Idaptive meshes are automatically produced in order to detect and resolve mild boundary layers and other sharp gradient situations. 


\section{PARTIAL DIFFERENTIAL EOUATIONS}

NAG

The Mark 7 edition of the NAG library has a new chapter devoted to this important problem area. At present it cuntains only four routines. However, later editions of the library promise to substantially increase the contents of this chapter.

\section{Elliptic}

The following seven codes comprise a suite of Fortran subroutines for the solution of Eliptic Partial Differential Equations. They were written by Paul Swartztrauber and Roland Sweet at the National Center for Atmospheric Research in Boulder, Colorado. The codes are very efficient and reliable and regarded as state-of-the-art software.

\section{*H 1 . PUSCRT *H S. S.}

Solves the Helmholtz equation in cartesian coordinates:

$$
\frac{\partial^{2} u}{\partial x^{2}}+\frac{\partial^{2} u}{\partial y^{2}}+\lambda u=f(x, y)
$$




\title{
w2. PUSPLR $=$ S. S.
}

Solves the Helnholtz equation in polar coordinates:

$$
\frac{1}{r} \frac{\partial}{\partial r}\left(r \frac{\partial u}{\partial r}\right)+\frac{1}{r^{2}} \frac{\partial^{2} u}{\partial \theta 2}+\lambda u=f(r, \theta)
$$

\author{
* 3. PUSCY *: S. S. \\ Solves the modified Helnholtz equation in cylindrical
}

coordinates:

$$
\frac{1}{r} \frac{\partial}{\partial r}\left(r \frac{\partial u}{\partial r}\right)+\frac{\partial^{2} u}{\partial z^{2}}+\frac{\lambda}{r^{2}} u=f(r, z) .
$$

This two-dimensional modified Helmholtz equation results from the Fourier transform of the three-dimensional Poisson equation.

$$
\text { w. L_PUSCSP :*: S. S. }
$$

Solves the modified Helwholtz equation in spherical crurdinates assuming axisymetry (no dependence on longitude):

$$
\frac{1}{r^{2}} \frac{\partial}{\partial r}\left(r^{2} \frac{\partial u}{\partial r}\right)+\frac{1}{r^{2} \sin \theta} \frac{\partial}{\partial \theta}\left(\sin \theta \frac{\partial u}{\partial \theta}\right)+\frac{\lambda}{r^{2} \sin ^{2} \theta} u=r(\theta, r)
$$

where $\theta$ is colatitude and $r$ is the radial coordinate. This two-dimensional modified Helmholtz equation results from the Fourler transform of the three-dimensional Poisson equation. 
W. PUSSSP wit S. S.

Solves the Helmholtz equation in spherical coordinates and on the surface of the unit sphere (radius of 1):

$$
\frac{1}{\operatorname{Sin} \theta} \frac{\partial}{\partial \theta}\left(\operatorname{Sin} \theta \frac{\partial u}{\partial \theta}\right)+\frac{1}{\operatorname{Sin}^{2} \theta} \frac{\partial^{2} u}{\partial \phi^{2}}+\lambda u=f(\theta, \lambda)
$$

where $\theta$ is colatitude and $\lambda$ is longitude.

\section{-16. POIS ES S. S.}

May be used to solve finite difference approximations to an equation of the form:

$$
a(x) \frac{\partial^{2} u}{\partial x^{2}}+b(x) \frac{\partial u}{\partial x}+c(x) u+\frac{\partial^{2} u}{\partial y^{2}}=g(x, y)
$$

\section{I. BLKTRI *W S. S.}

May be used to solve finite difference approximations to the general separable equation:

$$
a(x) \frac{\partial^{2} u}{\partial x^{2}}+b(x) \frac{\partial u}{\partial x}+c(x) u+d(y) \frac{\partial^{2} u}{\partial y^{2}}+e(y) \frac{\partial u}{\partial y}+f(y) u=g(x, y)
$$

Whenever possible POIS should be used in preference to BLKTAI. 


\section{Elliptic. Parabolic and Heperbolic}

Th: following three pieces of software, nanely PDEONE, MOL1D, and PDECOL, are each design $\geq d$ for solving coupled systems of nonlinear partial differen'isl equations of the form:

$$
\frac{\partial u}{\partial t}=\underline{f}\left(t, x, \underline{u}^{\prime} \underline{u}_{x}, \underline{u}_{x x}\right) \quad a \leq x \leq b \quad t \geq t_{0}
$$

where $\underline{u}$ is a vector of solution components

$$
\underline{u}=\left(u_{1}, u_{2}, \ldots, u_{n-1}, u_{n}\right),
$$

each of which is a function of the scalar quantities $t$ and $x$. The quantities $\underline{u}_{x}$ and $\underline{u}_{x x}$ are the corresponding vectors of partial derivatives

$$
\begin{gathered}
u_{x}=\left(\frac{\partial u_{1}}{\partial x}, \frac{\partial u_{2}}{\partial x}, \ldots \frac{\partial u_{n-1}}{\partial x}, \frac{\partial u_{n}}{\partial x}\right) \\
u_{x x}=\left(\frac{\partial^{2} u_{1}}{\partial x^{2}}, \frac{\partial^{2} u_{2}}{\partial x^{2}}, \ldots, \frac{\partial^{2} u_{n-1}}{\partial x^{2}}, \frac{\partial^{2} u_{n}}{\partial x^{2}}\right)
\end{gathered}
$$

and $\underline{f}$ is, in generil, a nonlinear function of the variables $x, \underline{u}$, $\underline{u}_{x}$ and $\underline{u}_{x x}$, with $n$ components, that is

$$
\underline{r}=\left(r_{1}, r_{2}, \ldots, r_{n-1}, r_{n}\right)
$$




$$
\underline{b}\left(\underline{u}, \underline{u}_{x}\right)=\underline{g}(t) \text {, }
$$

where $\underline{b}$ and $\underline{g}$ are vector valued functions with $n$ cosponents. The initial conditions assume that each solution component $u_{j}$ is a known function of $x$ at $t=t_{0}$, that is

$$
u_{j}\left(t_{0}, x\right)=\phi_{j}(x) \quad j=1, \ldots, n
$$

where $\phi_{j}(x)$ is a known function of $x$.

The numerical method that is used by PDCONE, MOL1D and PDECOL. is the method of ines. In this method the system of partial differential equations is converted into a system of first order ordinary differential equations, which is then solved using a sophisticated ODE integrator. The main difference between PDEONE, MOLID and PDECOL is the technique that is used to convert the PDE's into a system of ODE's. The codes that are likely to be more useful are PDECOL and MOL1D. The main reason for this is because the user has to interface an appropriate ODE integrator with PDEONE whereas the integrator is built into the packages PDECOL and MOL1D.

** PDEONE *E R. F. Sincovec and N. K. Madsen

This is an interface subroutine which uses centered finite difference approximations to convert one-dimensional systems of partial differential equations into a system of first order ordinary differential equations. The routine is intended to be 
used in conjunction with an appropriate ODE integrator. A suitable code for this purpose is GEARB.

The spatial differencing that is employed in PDEONE is nore suitable for paraboilc and elliptic partial differential equations than for hyperbolic ones. Therefore, users should exercise care when using PDEONE for hyperbolic systens.

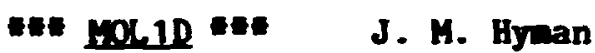

MOL ID is a fackage of subroutines for solving a systen of initial-boundai'y-value partial differential equations by the method of lines.

The system of P.X's that can be integrated must be of the form

$$
\frac{\partial u}{\partial t}=F\left(t, x, \underline{u}, u_{x x}, \underline{h}_{x}\right)
$$

where $\underline{h}$ is a vector

$$
\underline{h}=\left(h_{1}, h_{2}, \ldots, h_{n-1}, h_{n}\right)
$$

whose components are functions of $t, x, \mu, H_{x}$ and $\|_{x x}$, that is

$$
h_{j}=h_{j}\left(t, x, \underline{u}, \underline{u}_{x}, \underline{u}_{x x}\right), \quad j=1, \ldots, n .
$$

Like the previous subroutine MOLID uses finite differencis approximations to convert the system or PDE's into a system of first order ODE's. However, unlike PDEONE, it allows the user to 
PARTIAL DIFFEREITIAL EQUATIOHE

33

choose the spatial discretization that is tc be used. The available approximations are:

(i) Sixth order centered Jifferences.

(ii) Fourth order centered differences.

(iii) Second order centered differences.

(iv) Fast Fourier transform approximation.

(v) Fast Pourier transform with linear filtering of the higher wodes.

(vi) Second order differences oriented into the characteristics of $u_{t}=f_{x}$. Centered differences are used if the characteristics are perpendicular to the x-axis .

(vii) Bourth order centered differences or unsymmetric third order differences oriented into the characteristics of $u_{t}$ $=f_{x}$.

(viii) Derivative not to be calculated by the package.

Because this flexibility has been incorporated into the code, MOL 1D provides the user with the capability of solving a wide variety of different types of PDE's. This includes linear and nonlinear hyperbolic equations with or without discontinuities.

In order to solve the system cf first order ordinary differential equations, MOL1D uses GEARB. Therefore, the equations are integrated using either the implicit Adams methods or the backward differentiation formula methods. The user may specify which type of method is to be used. Alternatively the code will automatically select an appropriate method. 
PARTIAL DIFFERENTIAL EQUATIONS

MOL1D is a highly sophisticated piece of numerical software. It can accurately solve partial differential equations with a aininue of programing effort. Therefore, its use is highly recommended.

werer H. K. Madsen and R. F. Sincovec

This is a suite of subroutines for solving a systea of partial differential equations by the nethod of lines. It uses a finite element collocation procedure for converting the system of PDE's into a system of first order ODE's. The basis functions that are used in the collocation procedure are piecewise polynomials whose degree is specified by the user. The resultant system of ODE's is solved using a slightly modified version of GEARIB.

\section{-E MUGPK1 AE Arandt}

This is the complete set of codes from the ICASE Workshop on Multi-Grid Methods that was held in June 1978, at the MASA-Langley Research Conter in Hampton, Virginia. The codes include routines for solving the linear equations that arise from the finite difference approximation to the solution of certain types of partial differential equations. The method that is used to solve the linear equations is called a Multi-Level (or Multi-Grid) adaptive technique. This method may be viewed as one in which the partial differential. equation is discretized on a sequence of successively refined grids. The solution of the resillting linear equations from a coarse grid is then used to obtain the sulution 
on a finer grid. This process is repeated in an autonatic way from coarse grid to fine grid and vice versa. The resulting scheme proves to be very efficient for solving the discretized equations. Horeover, since the finer grids ay be introduced at any time in the solution process, it follows that the entire schene may be adaptive. Thus, for exanple, local grid refinenent near singular points or curved boundaries is possible.

In addition to the codes for solving the linear equations MUGPK 1, Iso contains routines for the grid refinement.

For further information about Muti-Grid methods, see the paper Multi-Level Adaptive Techniques (MLAT) for Partial Differential Equations: Ideas and Softwarem by Achi Brandt in the book Mathematical Softrare III by I. Rice, published by Academic Press in 1977, and/nr contact P. W. Gaffney. 


\section{MUPRRICAL DIFFBREATIATION}

Mat

\section{ne DOHAR EeE HAG}

At present there is only one subroutine, nanely D04MAF, in the NAG Iibrary for numer:cal differentiation. This is a seni-autonatic routine for obtaining approximations to the first 14 derivatives of a real valued function $f(x ;$ at a specified point $\dot{x}$. The user must provide a FUNC.TION subprogram for computing $f(x)$, and also a step lensth $h$ which is used in calculating the derivatives. It is because of this last requirement that Do4AAF is called a semi-automatic routine.

DO4AAF returns a set of approximations $\operatorname{DER}(j)$ and corresponding error estimates $\operatorname{EREST}(j)$ which hopefully satisfy

$$
\left|\operatorname{DER}(j)-f^{(j)}(\hat{x})\right| \leq \operatorname{EREST}(j), j=1, \ldots, n \leq 14,
$$

where $n$ is a user-specified upper limit on the number of derivatives required. Users interested in numerical differentiation should consult the fine introduction to the DO4 chapter in the MAG subroutine library manual, as well as the references cited therein. 


\section{INTEGRAL EOUATIONS}

MAG

- WE DOSME WEE MAG

This routine solves a linear nor-singular Fredholm integral equation of the second kind:

$$
f(x)-\lambda \int_{a}^{b} K(x, s) f(s) d s=g(x), \quad a \leq x \leq b
$$

where $f(x)$ is the unknowr function to be determined, $g(x)$ and the kernel $K(x, s)$ are known, and $K(x, s)$ is of 'split' type, that is, $K(x, s)$ is of the form

$$
K(x, s)=\left\{\begin{array}{ll}
k 1(x, s) & a \leq s \leq x \\
k 2(x, s) & x<s \leq b,
\end{array},\right.
$$

where $k 1$ and $k 2$ are well behaved functions while $k$ or its derivative with respect to $s$ may be discontinuous. D05AAF will also solve a linear non-singular Yelterca integral equation of the second kind:

$$
f(x)-\lambda \int_{a}^{x} x(x, s) f(s) d s=g(x), \quad a \leq x .
$$

$$
\text { - DOSABR *: NAG }
$$

This routine solves a linear non-singular Fredholm integral equation of the second kind with a smooth kernel. 


\section{INTEGRAL EQUATIOHS}

\section{8}

User's who wish to solve Integral equations are urged to consult the DO5 chapter in the NAG subroutine library manual. 
10. INTERPOLATION

NAG

HARUELL

IMSL

\section{-* SPLIHE/SEVAL *A: FTY}

SPLINE is a Fortran subroutine for computing the coefficients of a cubic spline

$$
\begin{array}{r}
s(x)=y_{i}+b_{i}\left(x-x_{i}\right)+c_{i}\left(x-x_{i}\right)^{2}+d_{i}\left(x-x_{i}\right)^{3} \quad x_{i} \leq x \leq x_{i+1} \\
i=1, \ldots, n
\end{array}
$$

which passes throtigh $n+1$ given values, that is

$$
s\left(x_{j}\right)=y_{j}, \quad j=1, \ldots, n+1 .
$$

The knots of $s(x)$ are at the data points

$$
x_{2}, x_{3}, \ldots, x_{n}
$$

This subroitine should be used only when it is sensible to approximate the data by a iunction wich passes through all of the function values.

SEVAL is a Function subprogram for evaluating the cubic spline function $S(x)$ at a specified value of $x$.

Because $S(x)$ has $n-1$ knots and because these knots must be at the given data points $x_{2}, x_{3}, \ldots, x_{n}$, the user c.snnot relax the continuity properties of $S(x)$. For excmrie, if it is known that the given values $\left\{y_{j}\right\} \quad j=1, \ldots, n+1$ are obtained from a function $y(x)$, say, which has a discontinuous first derivative in the range $x_{1} \leq x \leq x_{n+1}$, then it is not sensible to approxinate $y(x)$ by a cubic spine whose first two derivatives are continuous throughout 
$\left[x_{1}, x_{n+1}\right]$. It, follows that in sone cases the spline provided by SPLINE may be an unsatisfactory approximation to the data.

\section{-E IB06A/TG03A * Harwell (P. H. Gaffney)}

TB06A is a subroutine for computing a spline $R(x)$ of degree $k-1$ which passes through $n+1$ given values, that is

$$
\begin{aligned}
R\left(x_{j}\right)=y_{j}, & j=1, \ldots, n+1, \\
& 1 \leq k \leq n+1 .
\end{aligned}
$$

The knots of $h(x)$ are at the points

$$
n_{1}<n_{2}<\ldots<n_{n-k}<n_{n+1-k}
$$

where

$$
x_{i}<n_{i}<x_{i+k}, \quad i=1, \ldots, n+1-k .
$$

TBO6A should be used only when it is sensible to use an approximating function which passes through all of the given function values.

The advantages of TBO6A over, e.8., SPLIRE, are that the degree of the spline and the position of the knots may be specified by the user. This allows the user some flexibility in determining the continuity properties of the approximation.

TG03A evaluates a spline of degree $k-1$ and its derivatives $s^{(j)}(x), j=1, \ldots, n \leq k-1$, at a specified value of $x$.

WICUBIC * UCC-ND (P. W. Gaffney)

This is a package of subroutines for computing a bicubic spline function which interpolates values of a function $u$ : 


$$
\begin{aligned}
& u_{i j}=u\left(x_{i}, y_{i}\right) i=1, \ldots, n \\
& j=1, \ldots,
\end{aligned}
$$

on a rectangular grid.

The routines inplement the 1962 algorithm of de Boor for computing the coefficients of the spline.

The bicubic spline that is computed has knots at the interior grid points

$$
x_{2}, x_{3}, \ldots, x_{n-2}, x_{n-1}
$$

and

$$
\mathbf{y}_{2}, \mathbf{y}_{3}, \ldots, \mathbf{y}_{\mathrm{w}-2}, \mathbf{y}_{\mathrm{w}-1} \text {. }
$$

Therefore, it suffers from the same limitation as, for example, the one-dimensional spline obtained from SPLINE. 


\section{CURYE AND SURFACE FITTING}

\section{NAG}

HARUELL

IMSL

Some of the finest routines that exist at present for curve and surface fitting are in the E02 chapter of the NAG library. These routines are recommended without hesitation. Therefore, users with problems in this area are urged to consult the NAG library before anything else.

** IDRVIP/IDSEFT *N* Algorithm 526 in TOMS (H. Akima)

This is a subroutine package that implements Akima's method of bivariate interpolation and smooth surface fitting for irregularly distributed data points.

W. ADAPT * Algorithm 525 in TOMS (J. R. Rice)

Tnis is a subroutine for approximating a function of one variable, $f(x)$, to within a user-specified smoothness. It uses piecewise polynomials to approximate $f(x)$. The degree of the polynomials and the accuracy required of the approximation are 3ipfiled by the user. ADAPT also requires the user to supply a r:jir.s to calculace $f(x)$ and its derivatives. Therefore, ADAPT Is as:s:able for discrete data sets. 


\section{MINIMIZING OR GAXIMIZING A FUNCTION}

NAG

HARWELL

Computer users with problems in this area are strongly advised to consult the MAG and Harwell subroutine libraries, before using anything else. The software for this subject in the NAG library covers a wide variety of problems and reflects state-of-the-art methods. The corresponding software in the Harwell library can suffer from portability problems. However, like the NAG library, the software is extremely good and covers a significant range of problems.

\section{-WE LMDER1 *: MINPACK1}

This subroutine tries to minimize the sum of the squares of $m$ nonlinear equations in $n$ unknowns:

$$
\begin{gathered}
f_{1}\left(x_{1}, \ldots, x_{n}\right)=0 \\
f_{2}\left(x_{1}, \ldots, x_{n}\right)=0 \\
\vdots \\
f_{m}\left(x_{1}, \ldots, x_{n}\right)=0 .
\end{gathered}
$$

The user must supply a subroutine for computing the quantities

$$
f_{1}\left(x_{1}, \ldots, x_{n}\right), \quad 1=1, \ldots, m
$$

and the Jacobian matrix

$$
J A C=\left[\frac{\partial f_{1}}{\partial x_{j}}\right] \text {. }
$$


The numerical sethod that is used by LMDER1 is a codification of th: Levenberg-Marquardt algorithm. Because this athod is iterative, the user aust supply an initial estinate of the soluiion vector. In practice LADERI has proved to be relaitively insensitive to a poor initial estinate.

The documentation for LMDER1 is perfectly satisfactory and the user interface is excellent. Therefore, LMDER 1 can be used with confidence.

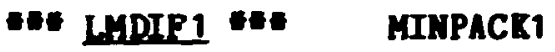

This subroutine tries to minimize the sum of squares of m nonlinear equations in $n$ unknowns:

$$
\begin{gathered}
f_{1}\left(x_{1}, \ldots, x_{n}\right)=0 \\
f_{2}\left(x_{1}, \ldots, x_{n}\right)=0 \\
\vdots \\
f_{m}\left(x_{1}, \ldots, x_{n}\right)=0 .
\end{gathered}
$$

The user must provide a subroutine which calculates the quantities

$$
f_{1}\left(x_{1}, \ldots, x_{n}\right), \quad 1=1, \ldots, m \text {. }
$$

The Jacobian matrix $\left[\frac{\partial r_{1}}{\partial x_{j}}\right]$ is then approximated, in LMDIF1, using finite differences.

$$
\text { Apart from the Jacobian calculation, LMDIF1 is sillar to }
$$
LMDER1. Therefore, it is recommended without reservation. 


\section{FUIN wa FTH (R. Brent)}

This is a function subprogram for computing an approxination to the point $x^{*}$, in the interval $a<x<b$, where $f(x)$ attains its minimum.

The numerical method that is used by FMIN is a combination of the golden-section search and successive parabolic interpolation. Therefore convergence to a minimum is guaranteed. However, if the function $f$ is not unimodal, then FMIN may converge to a local minimum which is not necessarily the global minimum.

\section{-He PRAXIS EE R. Brent}

This is a subroutine for minimizing a function of $N$ variables $f\left(x_{1}, x_{2}, \ldots, x_{n-1}, x_{n}\right)$

without calculating derivatives. It is a modified version of Powell's 19íl algoritnm, which uses conjugate directions to obtain the minimum. The main modification to Powell's algorithm is to the procedure for obtaining and maintaining linearly independent search directions. In PRAXIS this is accomplished by the principal axis method.

Numerical results indicate that PRAXIS is a very reliable subroutine for computing a minimum of a general function without calculating derivatives.

\section{* IFO2AD * Harwell (M. J. D. Powell)}

This is a modified version of Professor M. J. D. Powell's new routine VMCON for constrained optimization. Specifically VFO2AD is a Double Precision Fortran subroutine for calculating 
the least value of a function of several variables subject to general constraints on the values of the variables. The objective runction is

$$
F\left(x_{1}, x_{2}, \ldots, x_{n}\right)
$$

and the constraints are

$$
\begin{array}{ll}
c_{1}\left(x_{1}, x_{2}, \ldots, x_{n}\right)=0 & i=1,2, \ldots, p \\
c_{i}\left(x_{1}, x_{2}, \ldots, x_{n}\right) \geq 0 & i=p+1, \ldots, n
\end{array}
$$

where the $\left\{c_{i}\right\}$ ay be linear or non-linear functions of the variables $x_{1}, \ldots, x_{n}$.

The user is required to provide initial values for the variables $x_{1}, \ldots, x_{n}$, and suitable code for evaluating $F$ and $c_{i}$ and their first derivatives

$$
\frac{\partial F}{\partial x_{j}}, \frac{\partial c_{i}}{\partial x_{j}}, i=1, \ldots, m \text { and } j=1, \ldots, n
$$

The original nicoN was modified by J. K. Reid et al, so that it would be pontable and so that it would have a convenient user interface. With respect to the latter requirement VFC2AD, uses "reverse communication" for obtaining the values of

$$
F, c_{1}, \frac{\partial F}{\partial x_{j}} \text { and } \frac{\partial c_{1}}{\partial x_{j}}, 1=1, \ldots, m, j=1, \ldots, n \text {. }
$$

A description of the algorithm is given by M. J. D. Powell In $m_{A}$ Fast Algorithm for Nonlinearly Constrained Opifuization Calculations" which is published in the proceedings of the 
MINIMIZING OR MAXIMIZIMG a FUNCTION

47

Biennial Conference on Munerical Analysis, Dundee 1977, by Springer-Verlag. 


\section{MUTRIX OPERATIOUS}

\section{MAG}

\section{HARUELL}

IHSL

In this section we have included routines for the following problens:

$$
\begin{aligned}
& \text { Matrix Inversion } \\
& \text { Matrix Transformation }
\end{aligned}
$$

and

$$
\begin{aligned}
\text { Matrix Manipulation (for exauple: Transpose, } \\
\text { itultiplication, Subtraction, } \\
\text { Addition, etc.) }
\end{aligned}
$$

The routines for Matrix Inversion should not be used to solve simultaneous linear equations. 


\section{FIGENYN.UES AND EIGENYECTORS}

NAG

IMSL

HARHELL

\section{- WE EISPACK}

This is a collection of subroutines for computing the eigenvalues and/or the eigenvectors of a watrix. The types of matrices that can be handled by EISPACR are

COMPLEX GENERAL

COMPLEX HERMITIAN

REAL GENERAL

REAL SYMETRIC

REAL SYMMETRIC TRIDIACONAL

and SPECIAL REAL TRIDIAGONAL .

The subroutines in EISPACK are based on the algorithms published in the Springer-Verlag handbook series by Wilkinson and Reinsch. Therefore, users can be sure that the numerical methods employed by the subroutines are perfectly satisfactory. Moreover, since EISPACK is a product of the NATS (National Activity to Test Software) Project, the contents of EISPACK have been thoroughly tested and reflect qualitv mathematical software.

The corresponding subroutines in the 802 chapier of the NAG library are also based on the algorithms in the Wilkinson-Reinsch handbook. Moreover, the NAG routines are of the same high quality as those in EISPACK. Therefore, from a conventence point of view, 
computer users may prefer to choose the $\mathrm{F02}$ routines. This choice is recomended by the author.

1. LAHSO UCC-ND (D. S. Scott)

LANSO is a new subroutine for computing a specified number. of either the smallest or the largest eigenvalues, and corresponding eigenvectors, of a real symetric matrix $A$. It is designed for the case when the matrix $A$ is large. Consequently, for swall eigenproblems more appropriate routines are ayailable.

LANSO has been written in such a way that the user may take advantage of any sparsity pattern in the matrix $A$. Thus, it is particularly efficient for large sparse symetric matrices.

WA LANSOT UCC-ND (D. S. Scott)

This is a subroutine for computing all of the eigenvalues, and corresponding eigenvectors, of a real symetric matrix that are outside a given interval.

\section{W2. 12 Algorithm 496 in TOMS (L. Kaufman)}

This is a Fortran subroutine for computing the vector $x$ and the constant $\lambda$ such that the equation

$$
A \mathbf{x}=\lambda \mathbf{B x}
$$

is satisfied, where $A$ and $B$ are square matrices, which way be complex. The subroutine uses the LZ algorithm for finding $x$ and $\lambda$. Therefore, it may be used when either or both matrices are singular. 
15. DETERHINMNTS

NAG

IMSL

HARUELL 


\section{SIMULTANEOUS LINEAR EOLATIONS}

NAG

\section{HARWELL}

IMSL

The rc stines, in the NAG, Harwell and IMSL libraries, for solving systems of linear equations

$$
\underline{A x}=\underline{b}
$$

are among the best currently available. In particular the Harwell subroutine library contains the exceljent sparse matrix package MA28.

\section{*E: DECOMP/SOLVE WE FMM}

This is a pair of sutroutines for obtaining the solution of a system of linear equations of the form:

$$
\underline{A x}=\underline{b} \text {, }
$$

where $A$ is a real $n \times n$ matrix, $x$ is the vector of unknowns,

$$
x=\left(\begin{array}{c}
x_{1} \\
\vdots \\
x_{n}
\end{array}\right)
$$

\footnotetext{
and $\underline{b}$ is a vector of $n$ given components. Specifically, DECOMP performs Gaussian elimination, with partial pivoting, on the matrix $A$, and sives the multipliers and pivot information. It also provides an estimate of the condition of the matrix A. The size of this estimate indicates whether it is safe to try to complete the solution using SOLVE.
} 
Using the information obtained from DECOMP, subroutine SOLVE performs forward and back substitution to compute the solution $\underline{x}$ for any right-hand side $\underline{b}$. Both subroutines are excellent for analyzing and calculating the solution to a system of real linear equations when the matrix A does not possess any special structure, for example, $A$ is not banded. If the matrix does possess some structure, then it may be sensibie to use a routine that has been designed to take advantage of this structure.

\section{* LINPACK **}

This is a suite of subroutines for solving various types of linear systems. It contains routines for solving linear equations of the form

$$
\underline{A x}=\underline{b},
$$

where $A$ can either be a general square matrix or it can be banded, symmetric indefinite. symmetric positive definite, triangular or tridiagonal. There are also routines in LINPACK for rectangular matrices. Thus, linear least squares problems may be solved by using either the QR method or the singular value decomposition.

The subroutines in LINPACK are designed to be reliable, efficier.t and postable. Therefore, they are highly recomended.

*** Harwell (I. S. Duff)

This is a set of subroutines for the solution of sparse unsymmetric sets of linear equations:

$$
A \underline{x}=\underline{b} .
$$


The subroutines are divided into three distinct phases called Ma28A, Ma28B, and Ma28C. MA28 first permutes the matrix A into the block lower triangular form

$$
\left[\begin{array}{cccccc}
A_{1,1} & & & & \\
A_{2,1} & A_{2,2} & & & \\
A_{3,1} & A_{3,2} & A_{3,3} & & \\
\vdots & \vdots & \vdots & \cdots & A_{n, n}
\end{array}\right]
$$

where the matrices $A_{i, i}$ are square. Then, it performs Geussian elimination with pivoting on each diagonal block $A_{1, i}$ io obtain the factorization

$$
A_{i, i}=L_{i} U_{i} \quad i=1, \ldots, n
$$

where $L_{i}$ is a lower triangular matrix whose diagonal elements are equal to one, and $U_{i}$ is an upper triangular matrix.

The main advantage of permuting A into block lower triangular form is in the solution process. For, if the matrices $A_{i 1}$ are much smaller than $A$, (which is usually the case), then there is a saving in storage and execution time.

MA.28A provides the user with the option of omitting the permutation step and obtaining a full decomposition of the original matrix.

MA28B uses information from a previous call to Ma28A to factorize a new matrix which has a similar sparsity pattern.

MA28C solves sets of linear equations of the form 


$$
A x=\underline{b}
$$

or

$$
\mathbf{A}^{\mathrm{T}} \underline{\mathrm{x}}=\underline{\mathrm{b}}
$$

using the factorization obtained by MA28A or MA28B. The MA28 package is state-of-the-art software for this problem. It is reliable, efficient and fast. Therefore, it is recommended without reservation.

\section{* YSMPS/YSYPH *F: Yale}

These are two software packages for solving sparse systems of linear equations:

$$
\underline{A x}=\underline{b} .
$$

YSMPS solves the system when $A$ is symmetric.

YSMFN solves the system when $A$ is non-symmetric.

The numerical method that is used is a sparse variant of Gaussian elimination. However, no pivoting is performed for numerical stability.

For symmetric positive definite systems, that is when $A=A^{T}$ and all the eigenvalues of A are positive, YSMPS will probably be more efficient than MA28. However, for unsymmetric problems and for problems which require arme pivoting to maintain stability. users should use MA28 in preference to YSMPS or YSMPN.

\section{W. BT/BTMS WH: ACH LLL}

This is a pair of subroutines for solving linear equations

$$
\underline{A x}=\underline{b}
$$

where the matrix $A$ has tiae block tridiagonal structure 


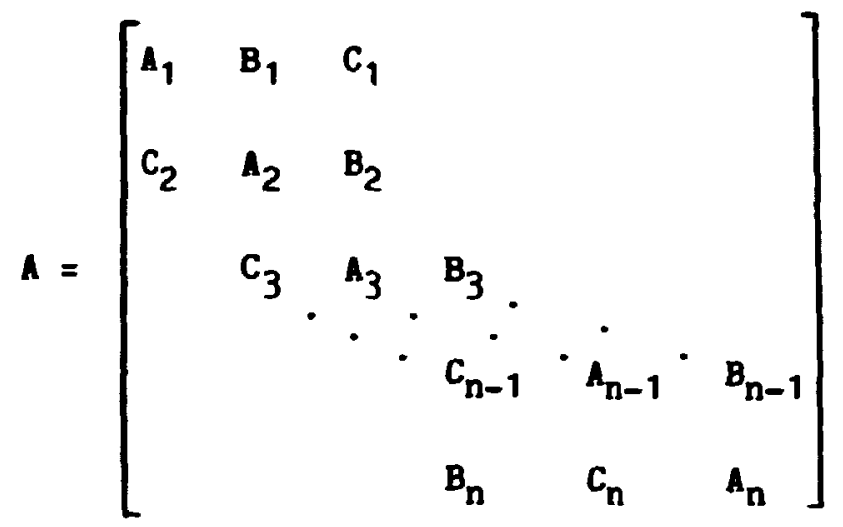

and each $A_{i}, B_{i}, C_{i}$ is a square matrix of order $m$.

Matrices of this type frequently occur in the numerical solution of systems of partial differential equations.

BT assumes that the matrix $A$ and the vector $\underline{b}$ can both be stored in core simultaneously. If this is not the case, then the minimal storage version of BT, namely BTMS should be used. This is the main difference between BT and BTMS.

The numerical method that is used is a block crout LU-decomposition of the matrix A followed by forward and back substitutions. The matrix $L$ is block lower triangular, and the matrix $U$ is block upper triangular with identity matrices on the diagonal. During the computation of the decomposition, partial pivoting is performed to maincain stability.

These routines are written in ANS Fortran. They have proved reliable and efficient, and they are duly recommended for block-tridiagonal systems of linear equations. 
*E. GMVGMS :E: Aigorithm 527 in TOHS (R. E. Bank)

These are two subroutines which are implementations of the generalized marching algorithm. They may be used to solve linear equations

$$
\underline{A x}=\underline{b}
$$

where the matrix $A$ is of the form

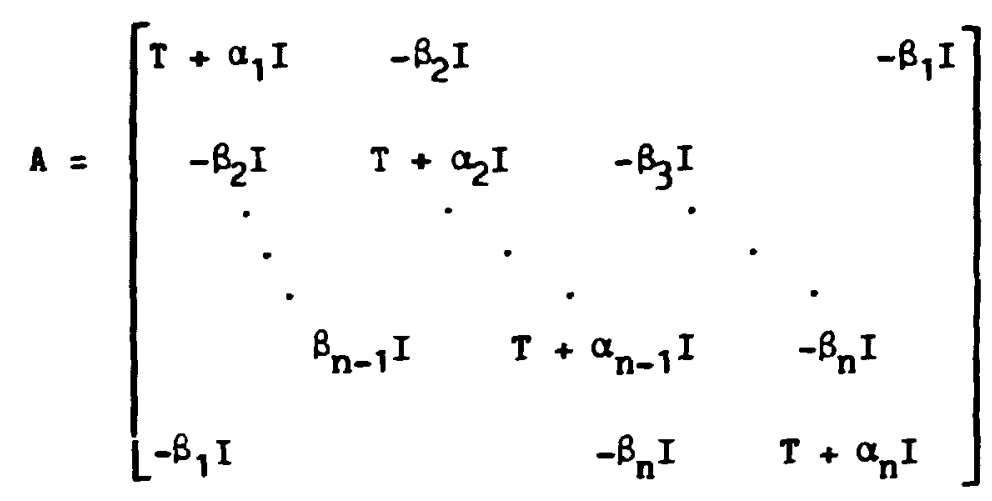

and $T$ is the nearly tridiagonal matrix

$$
T=\left[\begin{array}{ccccc}
\gamma_{1} & -\gamma_{2} & & & -\sigma_{1} \\
-\sigma_{2} & \gamma_{2} & -\sigma_{3} & \\
\ddots & \ddots \sigma_{m-1} & \gamma_{m-1} & -\sigma_{m} \\
& & & & \\
-\sigma_{1} & & -\sigma_{m} & & \gamma_{m}
\end{array}\right]
$$

with $\alpha_{1}, \beta_{1}, \gamma_{1}$, and $\sigma_{1}$ all scalars.

$$
\text { Linear equations of this form arise from 5-point }
$$

discretizations of separable or constant coefficient eliptic boundary-value problems on rectangular dowains. 
GH is appropriate for the case when the matrix $A$ is positivs: definite or negative definite.

When the natrix \& is positive or negative seniriefinite, (that is when all the eigenvalues of $A$ are respectively greater than or equal to zero, or less than or equal to zero), with zero an eigenvalue of multiplicity one, then subroutine Gus should be used to conpute a ieast squares solution.

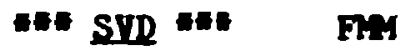

This is a subroutine for computing the singular value decomposition of a real rectangular matrix. It is a slightly modified version of the corresponding routine in EISPACK.

\section{-H: LAYSGK-HANSON CODES WH}

This is the complete set of main programs and subroutines from the nook "Solving Least Squares Problems" by C. L. Lawson and $\mathrm{R}$. J. Hanson. This is a very useful and practical book. The routines are well written and thoroughly documented, and they cover a wide range of problems. Consequently they are recommended for users who analyze and solve linear least squares problems. For the casual user, the appropriate routins in the MG library or LINPACK collection may prove more sati.ifactory.

* * CHEB *t: Algorithm 495 in TOMS (I. Barrodale and C. Phillips)

This subroutine is designed to compute the Chebyshev solution of an overdetermined system of linear equations: 


$$
\left[\begin{array}{cccc}
a_{1,1} & a_{1,2} & a_{1, n} \\
\vdots & \vdots & \vdots & \vdots \\
a_{n, 1} & a_{n, 2} & a_{n, n} \\
\vdots & \vdots & \vdots & \vdots \\
a_{m, 1} & a_{m, 2} & a_{m, n}
\end{array}\right]\left[\begin{array}{c}
x_{1} \\
\vdots \\
x_{n} \\
\vdots \\
x_{m}
\end{array}\right]=\left[\begin{array}{c}
b_{1} \\
\vdots \\
b_{n} \\
\vdots \\
b_{m}
\end{array}\right], \pm n
$$

Specifically, it computes a vector $X=\left[x_{1}, \ldots, x_{m}^{*}\right]^{T}$ which minimizes the quantity

$$
\underset{1 \leq i \leq m}{\operatorname{Max}}\left|b_{i}-\sum_{j=1}^{n} a_{i, j} x_{j}\right| .
$$

The subroutine may be used to solve the Chebysher data fitting problem:

Gizen $m$ data points $\left(z_{i}, y_{i}\right)$ ccmpute a function of the form

$$
p(z)=\alpha_{1} \phi_{1}(z)+\alpha_{2} \phi_{2}(z)+\ldots+\alpha_{n} \phi_{n}(z)
$$

such that the quantity

is minimized.

$$
\underset{1 \leq i \leq m}{\operatorname{Max}}\left|y_{i}-p\left(z_{i}\right)\right|
$$

If the values $y_{1}, \ldots, y_{m}$ contain only small inherent errors, then the Chebyshev approximation $p(z)$ may provide a more realistic approximation of the data than a least squares or a least-first-power fit. 


\section{STATISTICS}

IMSL

MAG

The IMSL subroutine library offers a wide selection of good software for statistical calculations. At present the MG library is weak in this area. However, there are plans to increase the number of statistics routines in later versions of the MaG library. 


\section{APPROXIMATION OF SPECIAL EUNCTIONS}

MAG

IMSL

\section{HARTELL}

The three subroutine libraries listed above are extremeIy weak in this area. Furthermore, there is a sad lack of software for special functions throughout the numerical software world. This unfortunate circumstance is likely to persist for sua time. Therefore, computer users who wish to compute special functions may have to resort to coding the approximations themselves. This is not such a difficult problem since there already exists some excellent software for approximating functions (see Sections 10 and 11). Therefore, the advice to users who require special function routines is to search $t$ :e mair. subroutine libraries, the routines described below, the routines in Appendices $C$ and $D$, and then, if they have not found the appropriate routine, to consult a Numerical Analyst.

\section{*. FUNPACK}

FUNPACK is a collection of quality routines for evaluating certain special functions. A list of the functions that may be approximated using FUNPACK is given below. This list highlights the fact that FUNPACK does not contain many routines. Moreover, it does not contain any routines which allow complex values of the independent variable. 


\section{Bunctions That Can be Appcoxinated Using FUIPACK}

1) Bessel functi ons of the first kind

$$
J_{0}(x) \text { and } J_{1}(x)
$$

2) Bessel function of the sesond kind

$$
I_{v}(x) \quad x>0,0 \leq v<1
$$

sonetines called Weber's function and sonetimes denoted by $\mathbf{H}_{\mathbf{v}}(\boldsymbol{x})$.

3) Modified Bessel functions of the first kind

$$
I_{0}(x) \text { and } I_{1}(x) \text {. }
$$

4) Zuvified Bessel functions of the second kind

$$
K_{0}(x) \text { and } k_{1}(x) \quad x \geq 0 \text {. }
$$

5) Dawson's Integral

$$
e^{-x^{2}} \int_{0}^{x} e^{t^{2}} d t
$$

6) Complete Elliptic integral of the first kind:

$$
\int_{0}^{\pi / 2}\left(1-k^{2} \sin \left(\phi^{2}\right)\right)^{-1 / 2} d \phi \quad 0 \leq k^{2}<1
$$

7) Corplete Elliptic integral of the second kind:

$$
\int_{0}^{\pi / 2}\left(1-R^{2} \sin \left(\phi^{2}\right)\right)^{1 / 2} d \phi \quad 0 \leq R^{2} \leq 1
$$


8) The exponential integral

$$
E i(x)= \begin{cases}\int_{-\infty}^{x} \frac{e^{t}}{t} d t & x>0 \\ -\int_{-x}^{\infty} \frac{e^{-t}}{t} d t & x<0\end{cases}
$$

9) The logarithmic derivative of the Gama function

$$
\psi(x)=\frac{\Gamma^{\prime}(x)}{\Gamma(x)}
$$

sonetimes called the Psi function. 


\section{MULTIPLE PRECISIQH ROLTIHES}

\section{*E HP Algorithe 524 in TOHS (R. Brent)}

MP is a collection of portable Fortran subroutines for performing multiple-precision floating point arithmetic, and for evaluating elementary and special functions in multiple precision. It is extrenely useful in applications that call for a higher precision than can be obtained using standard software. MP is state-of-the-art software and is highly recomended.

$A$ list of the contents of MP is given in Appendix D. 


\section{SOFTHARE TOOLS}

Strictly speaking, the information in this section does not pertain to numerical routines. However, because computer programmers should be aware of the tools in this section, we feel justified to inciuoe a description of the tools in this report.

\footnotetext{
* PFORT *i: Bell Laboratories

PFORT is a Fortran program for verifying that a Fortran computer code adheres to a portable subset of the American Hational Stanciard (ANS) Fortran (1966). The computer code may consist of a single subprogram, a group of subprograms, a main program, or a main program plus subprograms.
}

- *A DAYE University of Colorado

DAVE is a system for analyzing Fortran programs. It is designed to report the presence, or possible presence, of a wide variety of programmir errors. In addition it provides information on the usage of +11 local and global variables, and on the logical flow through a program. D!VE is written in Fortran and is designed for ease of portability.

1.: POLISH *I: University of Colorado

POLISH is an ANS Fortran program for editing Fortran programs. Specifically, POLISH, will read an ANS Fortran program and generate a copy of the program that is designed to be easy for a user to read. The copy of the origiral program is edited in such a way that statement labels are ordered, DO loops are 
indented and terminate on unique CONTINUE statements, and blank lines and unreferenced lioels are renoved. Apart frou these fixed editing features there are a number of others which may be controlled by the user in an automatic way. Thus, unlike some text editors, POLISH does not require the manual insertion of editing cues.

* BRNAML WE University of Colorado

BRNARL is a Fortran program for identifying basie blocks in a Fortran program. Specifically, BRHAML, will read an ANS Fortran program and generate a copy of the program which contains a subroutine call at the head of every basic n!nck. The name ard purpose of this subroutine are at the control of the user. Therefore, BRNAN may be used to gather statistics about the performance of a program. For example, the sections of code that are executed in a program may be recorded, the sequence in which statements are executed may be recorded, etc. 


\section{MESH GENERATORS}

WH TILE (P. J. Green and R. Sibson)

TILE is a group of ANS Fortran programs for computing the Dirichlet tessellation and the corresponding Delaunay triangulation from a given set of distinct data points. It is useful for finite element calculations, contour plotting, surface fitting, etc.

The main advant. $z E=$ of TILE over similar routines are that it is economical in storage and it is very fast. 


\section{APPENDIX A \\ Availability of Software}

The purpose of this appendix is to assist computer users, at Oak Ridge, in obtaining the software that is described in Section v. Sigure 1 describes the availability of the subroutine libraries: NAG, IMSL and HARWELL, and the software tools: DAVE, POLISH, BRNANL and PFORT. Figures 2 and 3 describe the commands that are required to invoke this software on the ORNL PDP-10, the FED Y-12 PDP-10 and the IBM 360 computers. In order to use the above software on the NMFECC computers, users are at the moment advised to contact Kirby Fong, telephone: (FTS) 8-532-4205. The remaining subroutines that are described in Section $v$ are available from the author on various pediums. Therefore, for one of these codes and for general numerical advice, computer users should not, hesitate to contact Pat Gaffney, telephone: 4-0630. 
ORNL. DWG 19.2502A

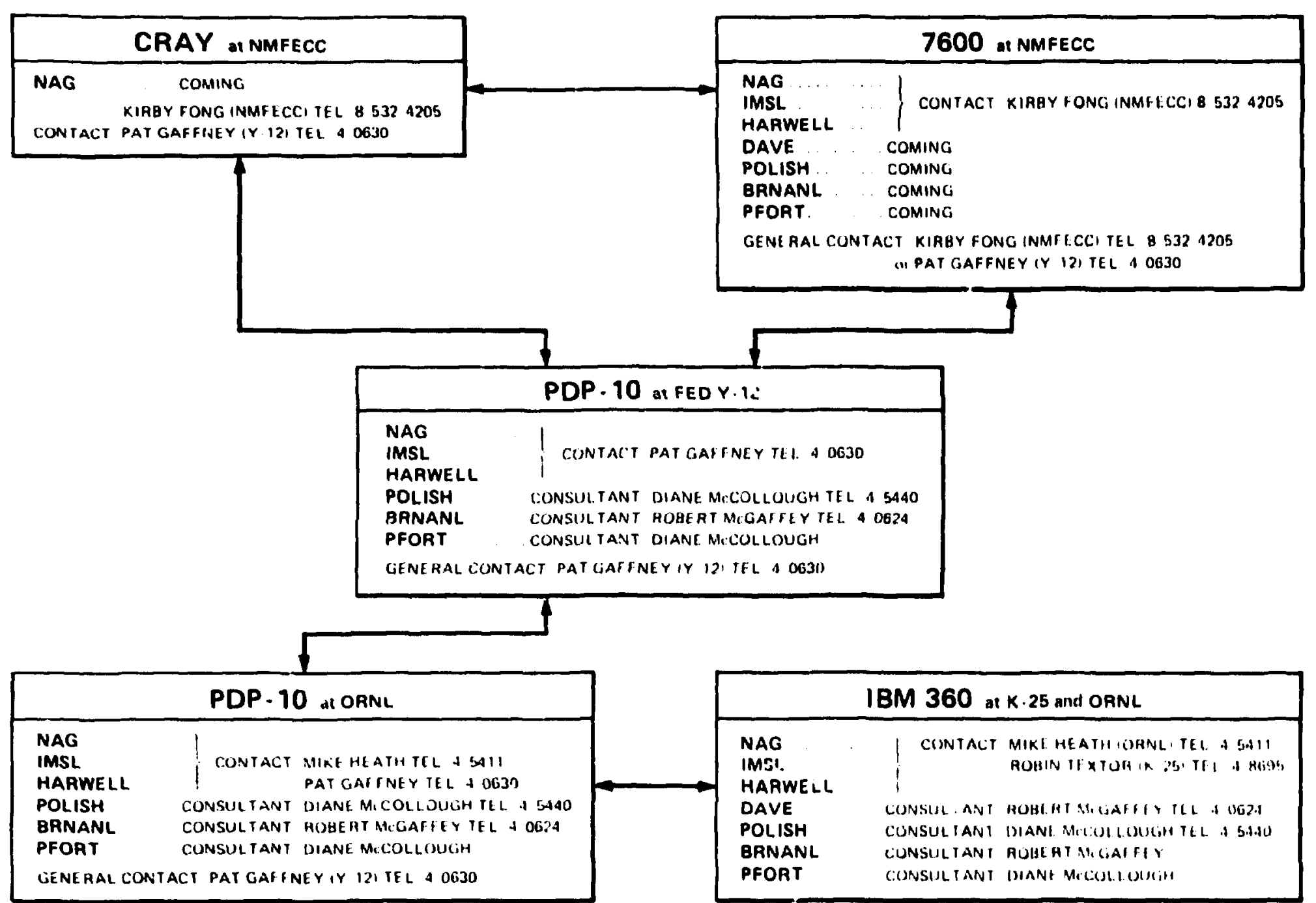

Figure 1. Avallability of Subroutine Libraries and Software Tools 
ORNL-OWG $79 \cdot 2615 \quad$ FED

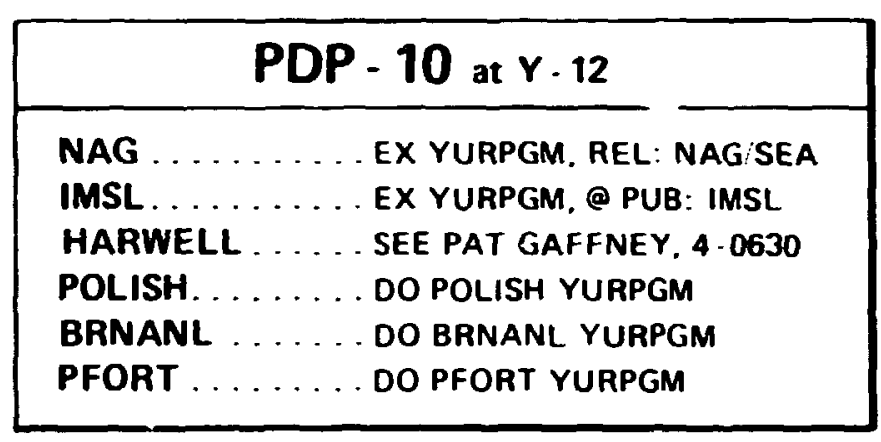

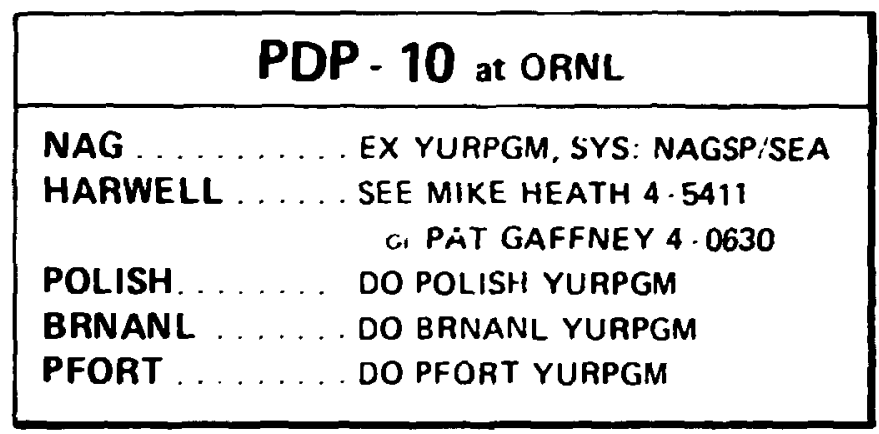

Figure 2. Commands Required to Invoke the Subroutine Libraries and Software Tools 
ORNL-DWG 79 2512R

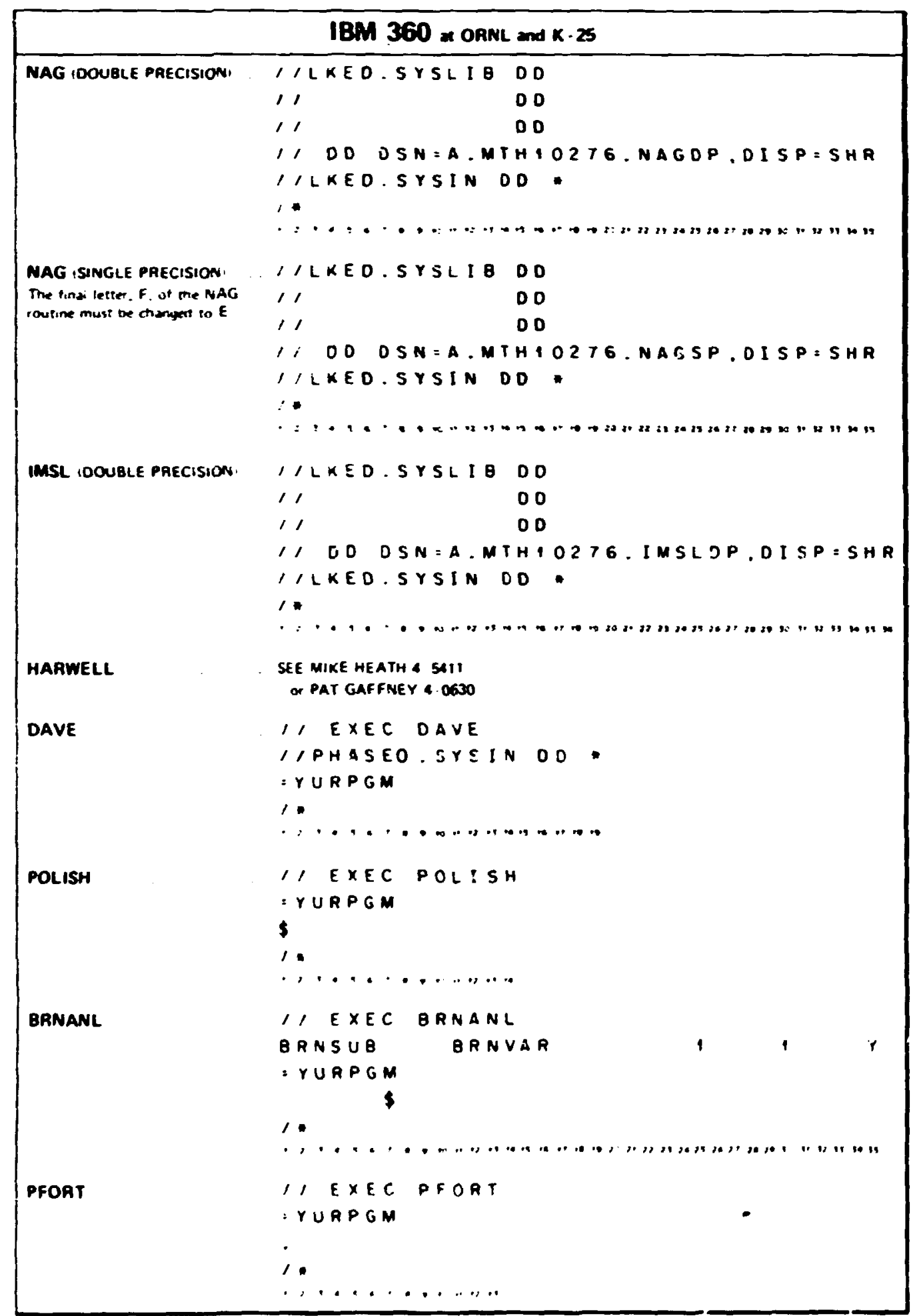

Figure 3. JCL Required to Invoke the Subroutine Libraries and Software Tools 


\section{APPENDIX B \\ The MASTI Database}

The purpose of this appendix is to describe a data base which contains information about the subroutines described in this paper. The name of the data base is NASTI which stands for Numerical Analysis Soitware Tutorial Inquicer. Thus, as the name implies MASTI is an interactive program. It is designed to inform computer users of some of the existing state-of-the-art software for particular problem areas. The areas that are covered by NASTI are listed in Section II of this paper, and in Figure 1 of this appendix. A user's manual for the NASTI data base is available as ORNL/CSD/M-97. Therefore, we will briefly describe the commands that are necessary to invoke NASTI.

The NASTI data base is managed by the System 1022 Data Base Management System. Therefore, we will use the nomenclature of this system to describe NASTI.

For each piece of software that is described in NASTI, there are six attributes, namely:

NAME

PURPOSE

PROBLEM

METHOD

ORIGIN

and VERSION •

"System 1022 Data Base Management System," Software House, Cambridge, Massachusetts 02138. 
The attribute

PROBLEA

is keyed.

COMPLEX ARITAMETIC

ZEROS OF POLYNOMIALS

ROOTS OF ONE OR MORE IRANSCENDENTAL EQUATIONS

SUMATIOH OF SERIES

QUA DRATURE

ORDIMARY DIFFERENTIAL EQUATIONS

PARTIAL DIFFEREATIAL EQUATIONS

NUIERICAL DIFFERENTIATION

INTEGRAL EQUATIONS

INTERPOLATION

CURVE AND SURFACE FITTING

MINIMIZING OR MAXIMIZIMG A FUNCTIOA

MATRIX OPERATIOHS

EIGENVALUES AND EIGENVECTORS

DETERMINANTS

SIMULTAKEOUS LINEAR EQUATIONS

STATISTICS

APPROXIMATION GF SPECIAL FUNCTIONS

MULTIPLE PRECISION ROUTINES

SOFTWARE TOOLS

MESH GEMERATORS

Figure 1. PROBLEY Areas for NASTI 
The remaining attributes are saic to be unkeyed.

The attribute that is most likely to be useful, for examining the data base is, PrOBLEy. Thus, in Figure 1, we give a list of the PROBLEM areas that are allowed by NASTI. A complete description of each attribute is given in the MASTI manual. Therefore, in order to describe how to use NASTI, we now consider a siople eranple.

\section{EXAHPLE}

Suppose that a computer user wishes to solve a partial differential equation. Furthermore, suppose that the user wishes to know some available state-of-the-art routines for this problem area. Then, the following sequence of commands could be used.

1) Log on to the PDP -10 at $Y-12$ or $X-10$.

2) Type the following:

R 1022

OPEN NASTI $[200,23000]$ at $Y-12$

or

OPEN NASTI $[6137,463]$ at $x-10$

FIND PROBLEM "PARTIAL DIFFERENTIAL EQUATIOHS"

The FIND command is designed for very fast retrieval of information from the data base. To accomplish this, it is restricted to the keyed attributes. Therefore, in this case it may be used with PROBLEM.

In the above example all of the records that are connected with the PROBLEM area PARTIAL DIFFERENTIAL EQUATIONS are selected. At this point the user way issue the command:

TYPE attribute ". . ." 
in wich case the attribute "..." for every record that has been selected, by the FIND comand, will be typed at the user's terainal. To avoid having to retype every attribute, the user can issue the comand

$$
\begin{array}{ll}
\text { USE NASTI .TYP }[200,23000] & \text { at } Y-12 \\
\text { USE NASTI.TYP }[6137,463] & \text { at X-10 }
\end{array}
$$

The file MASTI.TIP is designed so that all of the attributes, for every record that has been selected, will be typed at the user's terminal. If the user requires this information to be sent to a disk file, then the command

$$
\begin{array}{ll}
\text { USE NASTI.FIL }[200,23000] & \text { at } Y-12 \\
\text { USE NASTI.FIL }[6137,463] & \text { at X-10 }
\end{array}
$$

will write the information in the file NaSTI.OUT. As noted above the FIND command may only be used with the keyed attributes. For the unkeyed attributes the SEARCH commend (which must follow a FIND command) may be used. For example the sequence of commands in 2) may be replaced with

R 1022

OPEN MASTI $[200,23000]$

FIND PROBLEM "PARTIAL DIFFERENTIAL BQUATIONS"

SEARCH PURPOSE CONTAIMS "YOISSON"

In this case the FIND command selects all of the recoros for the problem srea of partial differential equations. Then, the search command:

SEARCH PURPOSE CONTAINS "POISSON" selects from this group all of the records whose a':tribute PURPOSE 
contains the word Poisson. The operator CoNTAINS way be abbreviated to $\mathrm{CT}$.

The above examples are intended only to introduce users to the NASTI data base. In order to realize the full potential of the 1022 system, users are urged to consult the system 1022 manual. 


\section{APPENDIX C}

\section{SANDIA Special Function Routines}

This appendix contains a list of subioutines, from the Sandia Mathematical Subroutine Library, for the approximation of special functions .

The information contained in this appendix appears in the Sandia report SANDT7-1441 entitled: "Brief Instructions for Using the Sandia Mathematical Subroutine Library (Version 7.2)' by Karen H. Haskell and Randall E. Jones.

\section{Bessel Functions}

BESI for $I_{a}(x)$, where $x$ is any nonnegative number, $x=0$

BESJ for $J_{\mathcal{U}}(x)$, where $\alpha$ is any nonnegative number, $x \geq 0$

BESKN for $K_{n}(x)$, where $n$ is any nonnegative integer, $x>0$

BESYN for $Y_{n}(x)$, where $n$ is any nonnegative integer, $x>0$. These routines can also return $N$ member sequences $I_{x+k}(x)$, $J_{a+k}(x), K_{n+k}(x), Y_{n+k}(x), k=0,1, \ldots, N-1$.

The firy funct: ons are also available through the routines

AIRY for $A i(x)$

DAIRY for $\mathrm{AI} \cdot(\mathrm{x})$

BAIRY for $B i(x)$

DBAIFY for $\mathrm{BI}^{\prime}(x)$.

The Hankel functions $H_{n}^{(1)}(x)$ and $H_{n}^{(2)}(x)$ can be evaluated by means of

$$
\begin{aligned}
& H_{n}^{(1)}(x)=J_{n}(x)+i Y_{n}(x) \\
& H_{n}^{(2)}(x)=J_{n}(x)-i Y_{n}(x) .
\end{aligned}
$$




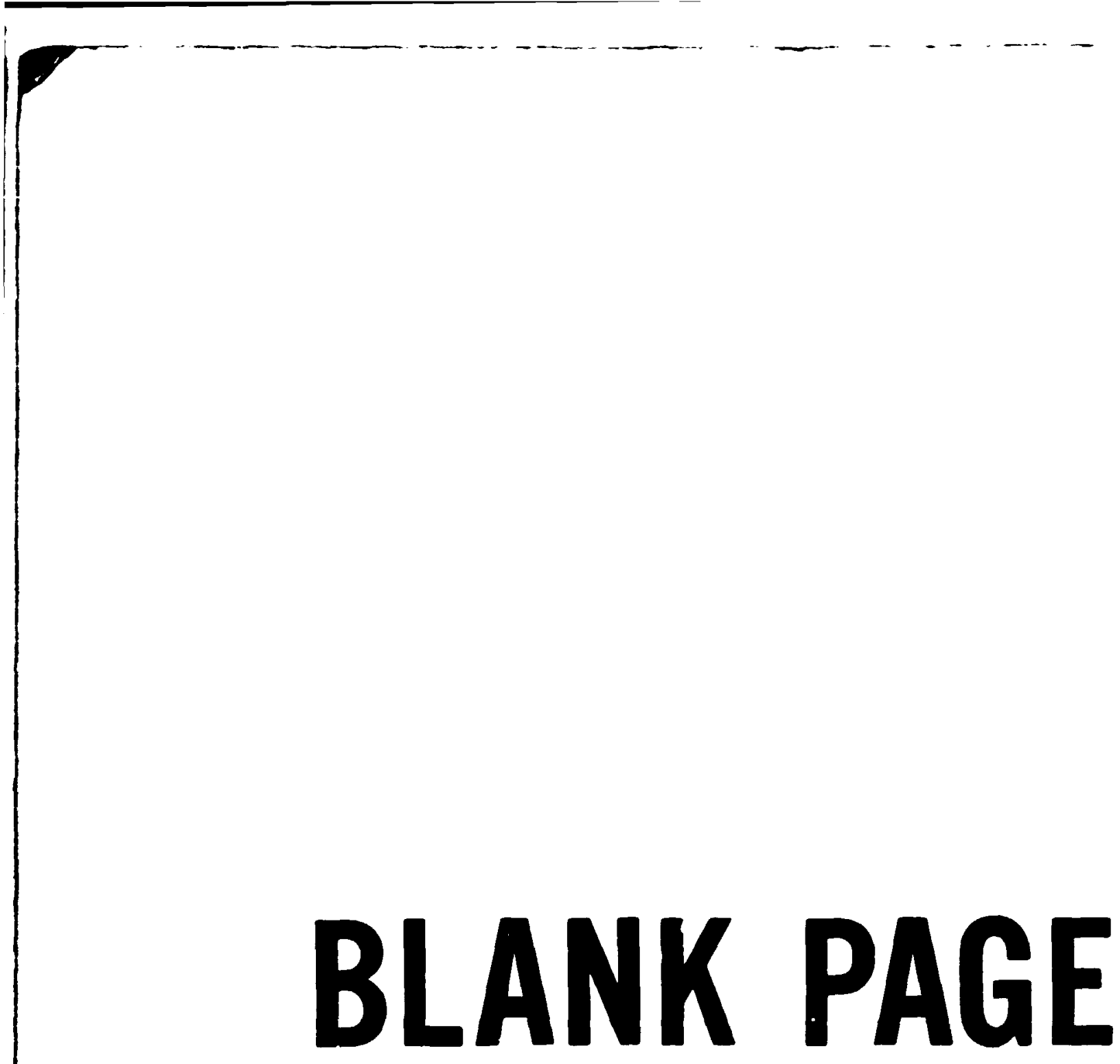


All Bsssel function routines produco near-maximum accuracy. That is, they are nearly as accurate as the systen Sj:! and CoS routines in the oscillatory regions, and nearly as accurate as EXP in the nonoscillatory regions. All routines are easy to call and do not require any working storage. Many of the routines provide scaling options to extend the admissible argument ranges.

\section{Beta Functions}

BETALN computes the logarithm of the beta function

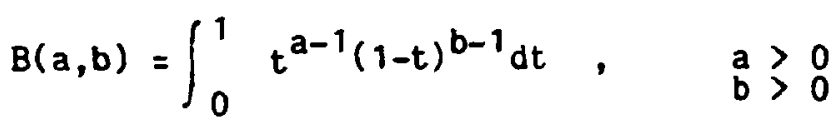

for positive $a$ and $b$. This function can also be expressed in terms of gamma functions

$$
B(a, b)=\frac{\Gamma(a) \Gamma(b)}{\Gamma(a+b)}
$$

but the routine has been constructed to minimize logarithm computation .

BETAIC and BETBIC both compute tre incomplete beta function

$$
I_{x}(a, b)=\frac{1}{B(a, b)} \int_{0}^{x} t^{a-1}(1-t)^{b-1} d t \quad \begin{array}{ll}
a \geq 0 \\
x \geq 0
\end{array}
$$

for nonnegative $x$, where $I_{x}(a, b)$ has been normalized by $B(a, b)$ so that $) \leq I_{x} \leq 1$. The difference between BETAIC and BE 3 BIC is that $N$ nember sequence. $I_{x}(a+k, b)$, indexed on the parameter $a$, an be computed with BETAIC while $N$ member sequences $I_{x}(a, b+k)$, indexed on the parameter $b$; car be computed with BETBIC, $k=0,1, \ldots, N-1$. 
If absolute accuracy is acceptable, 'he argument $x$ for $I_{x}(a, b)$ can be kept less than or equal to $1 / 2$ by using the identity

$$
I_{x}(a, b)=1-I_{1-x}(b, a)
$$

when $x>1 / 2$. This makes the evaluation several times faster than direct evaluation of $I_{x}(a, b)$ when $x>1 / 2$.

\section{Gamma Functions}

GAMFN evaluates the gamma function

$$
\Gamma(x)=\int_{0}^{\infty} e^{-t} t^{x-1} d t, x>0
$$

and its analytic continuation for $x<0$ for $-169<x \leq 170$ except at the poles $x=0,-1,-2, \ldots$ The gamma function is related to the factorial of $x$ (when $x$ is an integer) by

$$
x 1=\Gamma(x+1) \text {. }
$$

However, many applications involve multiplying or dividing gamma functions by other functions which partially cancel the fast growth of $\Gamma(x)$. In these cases logarithms are appropriate and one should use GAMLN for in $\Gamma(x), x>0$ for better scaling which will extend argument ranges.

GAMMAZ evaluates the gamma function for complex arguments 2 except in the neighborhood of the poles, $z=0,-1,-2, \ldots$ GAMIC and GAMTL compuce the incomplete gamma functions 


$$
\begin{array}{ll}
Y(\alpha, x)=\frac{1}{\Gamma(\alpha)} \int_{0}^{x} e^{-t} t^{\alpha-1} d t & \\
\Gamma(\alpha, x)=\frac{1}{\Phi(\alpha)} \int_{x}^{\infty} e^{-t} t^{\alpha-1} d t &
\end{array}
$$

respectively for positive $\alpha$ and nonnegative $x$. These functions have been normalized by $\Gamma(\alpha)$ so that $0 \leq \gamma<1,0<\Gamma \leq 1$ and $\gamma(\alpha, x)+\Gamma(\alpha, x)=1$. 


\section{APPENIIX D \\ The Contents of MP (see Section 19)}

This appendix contains a list of those subroutines in the multiple precision package MP, which may be useful to computer users. In the list below an "MP number" means a multiple-precision number.

MPABS Computes absolute value of an MP number

MPADD Adds two MP numbers

MPADDI Adds an MP number to an integer

MPADEQ Adds a rational number to an MP number

MPART 1 Computes ARCTAN (1/N) for N.GT. 1 (called by MPPI)

MPASIN Computes ARCSIN of an MP number

MPATAN Computes ARCTAN of an MP number

MPBERN Computes BERNOULLI numbers B2, $34, B 6, \ldots$

MPBESJ Computes Bessel function $J(N U, X)$ for MP $X$ and small integer NU

MPBES2 Routine called by MPBESJ (uses backward recurrence to evaluaie $J(N U, X)$ )

MPCDM Converts double-precision to multiple-precision

MPCIM Converts integer to multiple-precision

MPCLR Sets some digits of an MP number to zero

MPCMD Converts an MP number to double-precision real

:APCMDE Converts an MP number to (double-precision) fraction and (decimal) exponent

MPCMEF Converts MP number to fraction and (decimal) exponent

MPCMF Finds fractional part of an MP number

MPCMI Convert3 an MP number to an integer

MPCMIM Converts an MP number to a muitiple-precision integer 


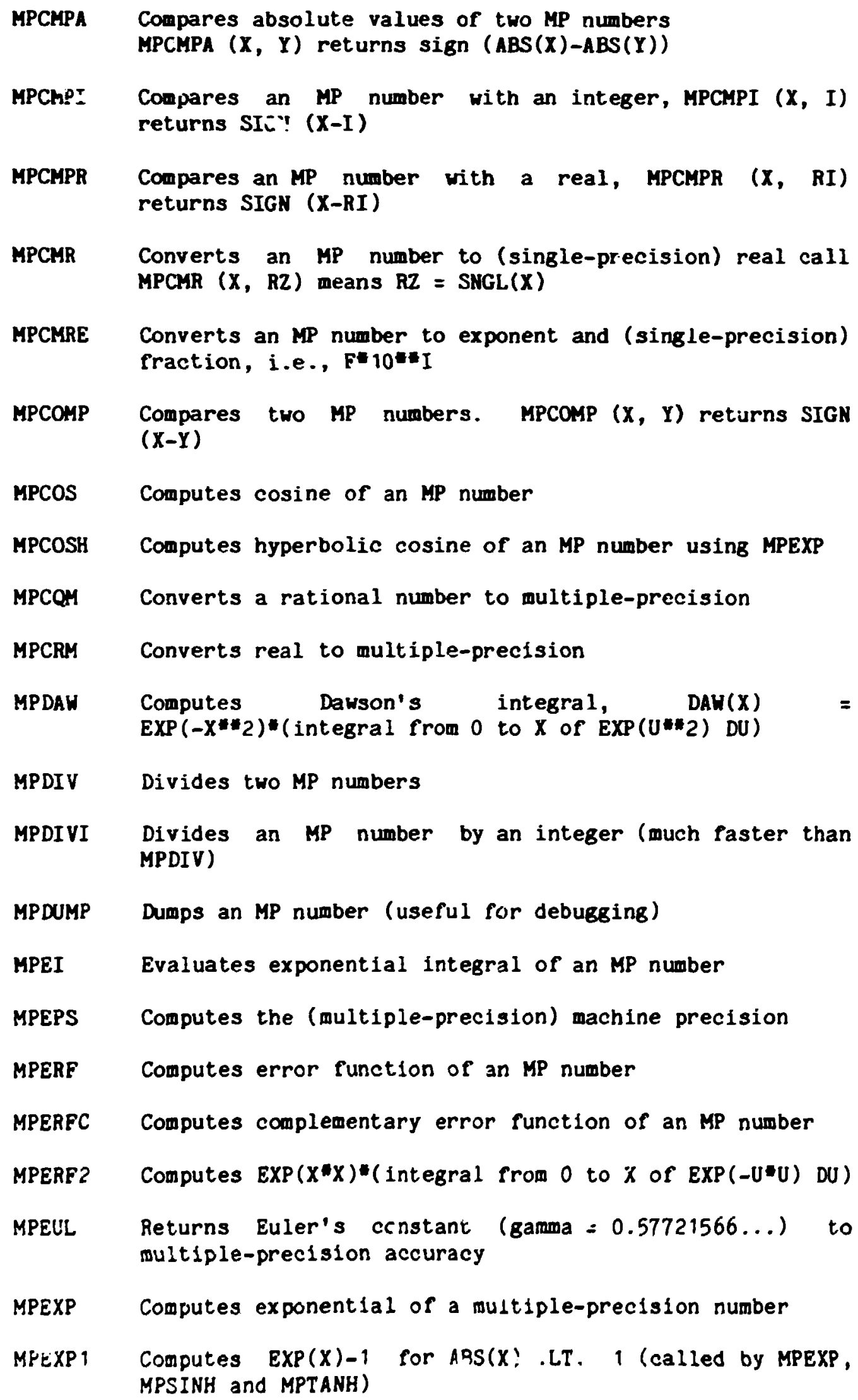


MPGAM Computes gamma function of an MP argument

MPGAMQ Computes gamma function of a rational argument

MPGCD Divides two integers by their greatest common divisor

MPHANK Routine called by MPBESJ (evaluates Hankel's asymptotic series for Bessel functions)

MPIN Converts fixed-point number read under $A 1$ format to multiple-precision

MPINE Same as MPIN but result is multiplied by a power of 10 (useful for reading in floating-point numbers)

MPLI Evaluates logarithmic integral LI(X)

MPLN Computes natural 108 of an MP number

MPLNGM Computes LN(GAMMA $(X))$ for positive MP $X$, using Stirling's approximation

MPLNGS Computes natural $\log$ of an MP number, using the Gauss-Salamin algorithm. Recommended for testing MPLN and MPLNI only.

MPLNI Computes natural $\log$ of an integer

MPLNS Computes $L N(1+X)$ for small MP $X$

MPL235 Computes natural log of an integer whose prime factors are 2,3 and/or 5 (called by MPLNI)

MPMAX Computes the maximum of two MP numbers

MPMAXR Computes the largest positive MP number

MPMIN Computes the minimum of two MP numbers

MPMINR Returns the smallest normalized positive MP number

MPMUL Multiplies two MP numbers

MPMULI Multiplies an MP number by an integer (faster than MPMUL)

MPMULQ Multiplies MP number by a rational number

MPNEG Reverses sign of an MP number

MPOUT Converts multiple-precision to a form suitable for printing under A1 format (corresponds to $F$ or I formats) 


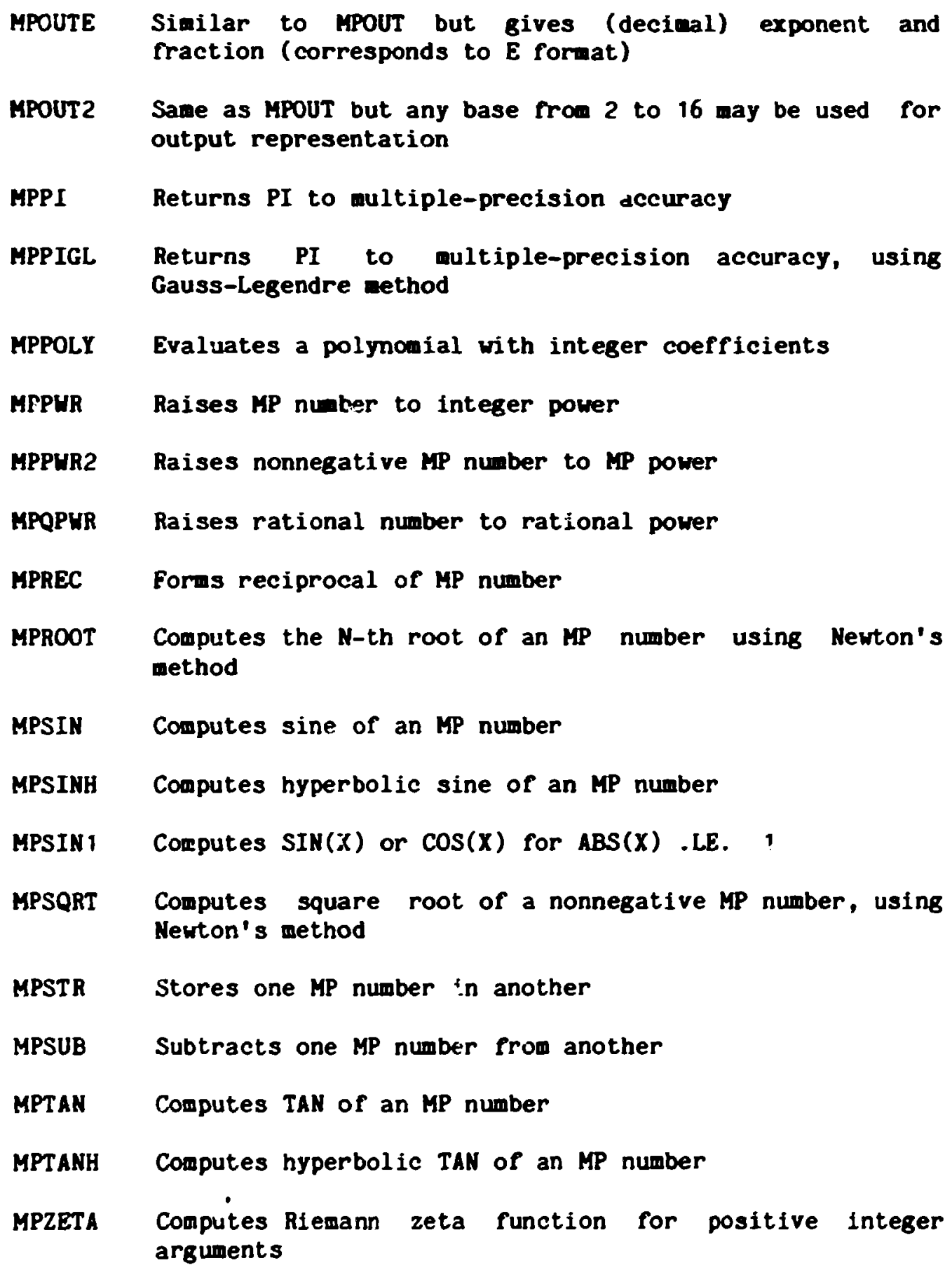

